\title{
Nutzerbasierte Bewertung von Büroimmobilien
}

\author{
Eine Post-Occupancy Evaluation auf Basis \\ umweltpsychologischer Aspekte unter besonderer \\ Berücksichtigung von Zufriedenheit, Gesundheit und \\ Produktivität
}

\section{Dirk Krupper}

Online publiziert: 7. Juli 2015

(c) Die Autor(en) 2015. Dieser Artikel ist auf Springerlink.com mit Open Access verfügbar.

Zusammenfassung Das Management von Büroimmobilien beschränkt sich meist auf die Reduktion der Raumkosten. Dabei findet typischerweise keine Berücksichtigung des Trade-off zwischen Arbeitsplatzkosten und Mitarbeiterproduktivität statt. Der vorliegende Aufsatz greift dieses Problem auf und untersucht die Zusammenhänge zwischen Büroumgebung und deren Wirkungen auf Nutzer. Zunächst werden theoretische Erkenntnisse zu einzelnen Effekten auf Zufriedenheit, Leistungsfähigkeit und Gesundheit aus dem Forschungsfeld der Umweltpsychologie zusammengeführt und in einem Rahmenmodell aufgezeigt. Darauf aufbauend wird anhand einer empirischen Untersuchung die simultane Betrachtung solcher Beziehungen mittels einer Kausalanalyse dargelegt. Die Ergebnisse stellen die Bedeutung einer adäquaten Büroumgebung für den Erfolg sowohl des einzelnen Mitarbeiters als auch der ganzen betrieblichen Organisation heraus. Das Potenzial zur Steigerung der Leistung lässt sich für die vorliegende Erhebung im Mittel auf $20 \%$ beziffern und stellt somit die einseitigen Bestrebungen von Kosteneinsparungen im Immobilienmanagement grundsätzlich in Frage. Als wesentlicher Baustein einer standardisierten Bewertung wird zudem das Konzept der Post-Occupancy Evaluation vorgestellt, welches Leitlinien zum Abgleich von Immobilie und Nutzeranforderungen bietet. Schließlich werden auch Integrationsmöglichkeiten von nutzerorientierten Bewertungen in das Management von Büroimmobilien als betriebliche Ressource aufgezeigt.

Schlüsselwörter Büroimmobilie · Immobilienportfolio · Umweltpsychologie · Stress

D. Krupper $(\bowtie)$

Commerz Real Investmentgesellschaft $\mathrm{mbH}$,

Friedrichstrasse 25,

65185 Wiesbaden, Deutschland

E-Mail: dirk.krupper@commerzreal.com 
Abstract Since there are millions of office workers spending $8 \mathrm{~h}$ the day for half of their life time in their office it should be obligatory to evaluate the impact of workspace and building on employee's satisfaction, performance, and healthiness. But today organizations are focused only on saving real estate costs, although employee costs are around 8-10 times higher than building costs. Actually, based on typical goals of organizations like growth, performance, productivity, sustainability, our perspective rather should be turnover or profit per person which is again typically much higher than employee cost. The questions to answer is how office buildings are affecting people's behavior and what consequences are to be expected. Knowledge of the relationship between people and the build environment is existing in theory and research in fields and content areas of environmental psychology. A lack of assessing office buildings from a user perspective is even more surprising because these studies are present for decades and should be part of research in real estate and architecture for years.

A systematical approach to evaluate the performance of buildings in terms of the requirements of building occupants including productivity, comfort and satisfaction is the so called Post Occupancy Evaluation (POE). Measuring fitness for use is not only assessing technical performance and sustainability of building elements. Besides assessing overall building quality the most important part of such an evaluation is getting feedback from users of the specific building or being more precise-from each user group. Type of job, workflows, and schedules will differ from each office worker as well as psychosocial factors, such as social support from colleagues and supervisor, job decision latitude, job demand, and time pressure.

The present paper is offering a framework of evaluation criteria based on multiple studies of environmental behavior, organizational behavior, an empirical study testing the theoretical model, and steps for integrating a POE in building and portfolio analysis of an organization to enhance performance of office workers. Data for the study were obtained from an evaluation of 11 office buildings. More than 630 office users participated the survey, corresponding an exceptionally high response rate of $41.7 \%$. Results highlight a significant relationship between office environment and job satisfaction, job performance, and healthiness. The potential boost of office workers performance is $20 \%$ on average based on the perceived negative impact of building environment. Spatial conditions like size of workspace, size of office space, and privacy is as important as physical conditions like climate, air quality or noise. Having control over environmental and spatial conditions is one of the major items in terms of being satisfied with the building conditions in total. Compared to all office conditions conference rooms and spatial conditions for face to face communication are least important even to those office workers how spent most of their time in meetings. This finding is of special interest since one of the main arguments for implementing open space concepts is improving ease of communication between workers and teams. Having a lack of environmental conditions in open plan offices in mind, the present study indicates a negative tradeoff between concepts of cell offices and open space even considering an improvement in communication. The study included a questionnaire to managers for data triangulation in order to check whether evaluation of staff is predictable by their supervisors. Results indicate that managers were not able to predict personnel's assessment, so 
ideally users should be involved in the decision process when their office space is affected.

For an in-depth analysis users were clustered into different groups based on their job activities and based on their individual characteristics. Independent of user's allocation to one of the clusters there is some general findings. Absent of noise, size of individual office space and ability to concentrate on tasks is most important to all office workers. Depending on user groups, social support from colleagues and supervisors can influence satisfaction with the office environment. Obviously users that are unhappy with the job situation project their dissatisfaction onto the office environment. Even control on job tasks and job demand may have an impact on perceived satisfaction with office and building environment, depending on user clusters.

The results of the present empirical study give strong support to establish Post Occupancy Evaluation as an instrument to enhance performance of office users, rather than simply save costs in real estate and ignore the relationship between building, business, and behavior. Obstacles still remain because in most organizations savings are achievable in short term through reorganization of space and renegotiations of lease term contracts or moving into another office building. Such an implementation of POE, user feedback, and user participation rather means a paradigm shift, gaining higher turnover and higher results on the long run.

Keywords Post occupancy evaluation · Performance $\cdot$ Environmental psychology $\cdot$ Stress

\section{Büroimmobilien als betriebliche Ressource}

In Deutschland liegt die Anzahl der Bürobeschäftigten bei schätzungsweise 12,5 Mio. Menschen (Bulwien et al. 2008). Die existierende Büromietfläche wird dabei auf über 320 Mio. $\mathrm{m}^{2}$ geschätzt (Bulwien et al. 2008). ${ }^{1}$ Diese Bürofläche wird als Arbeitsumgebung für die Büroarbeit zur Verfügung gestellt. Wird vereinfacht von einem achtstündigen Arbeitstag an geschätzten 220 Arbeitstagen im Jahr über einen Zeitraum von 35-40 Arbeitsjahren ausgegangen, verbringen Büromitarbeiter einen Großteil der täglichen Wachzeit und darüber hinaus ca. die Hälfte ihres Lebens in dieser Arbeitsumgebung. Durch diverse Studien wird die Relevanz dieser Büroumgebung als betriebliche Ressource für Zufriedenheit, Leistungsfähigkeit und Gesundheit der Mitarbeiter belegt (Moleski und Lang 1986, S. 8; Marans und Spreckelmeyer 1986; Ferguson und Weisman 1986; Sutton und Rafaeli 1987, S. 269; Carlopio 1996, S. 337; Wells 2000, S. 250; Feige et al. 2013). Ausgehend von diesen Beziehungen zwischen Nutzer und Immobilie lässt sich erwarten, dass die Qualität der Arbeitsumgebung aufgrund ihrer Bedeutung für Mitarbeiter und Organisationen einer regelmäßigen Kontrolle unterzogen wird. Zweck dieser Prüfung muss ein kontinuierlicher Abgleich auf Passung zwischen den Nutzerbedarfen und der Büroumgebung sein, um optimale Arbeitsbedingungen zu schaffen.

${ }^{1}$ Flächenangabe in MF-G nach gif. 
Abb. 1 Kostenrelationen eines Büroarbeitsplatzes am Beispiel Banken und Finanzdienstleistungen für den Standort Frankfurt

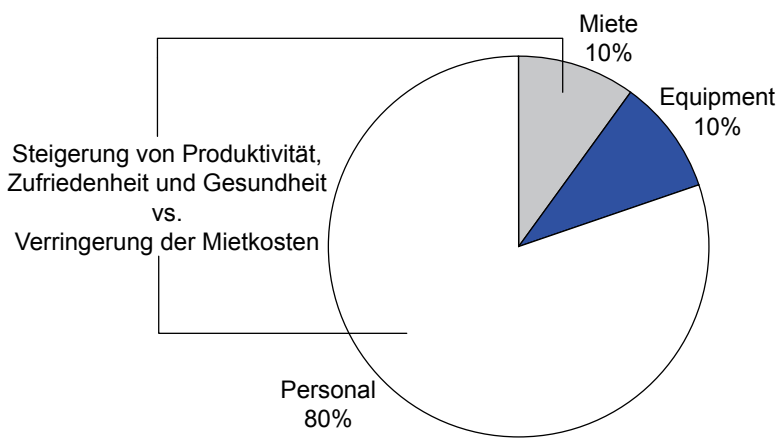

Erstaunlicherweise findet aber eine standardisierte Überprüfung und Bewertung der Einflüsse auf die Büronutzer nicht statt, obwohl in der Fachliteratur z. B. auf erzielbare Steigerungen der Produktivität der Mitarbeiter zwischen 5\% bis 50\% verwiesen wird, welche sich durch Anpassungen einzelner Umgebungsbedingungen erzielen lassen (Brill et al. 1984, S. 57; Brill et al. 2001, S. 18; Gifford 2007, S. 411; Pfnür und Weiland 2010, S. 1). ${ }^{2}$ Unternehmen scheuen häufig Investitionen in die Verbesserung der Arbeitsumgebung aufgrund der ohnehin hohen Kosten für qualifizierte Mitarbeiter, Büroflächen und Equipment. Der Fokus liegt vielmehr auf der Betrachtung der reinen Mietkosten pro Arbeitsplatz und deren Senkung, weniger im Management der materiellen Ressource Büroumgebung (Bon 1989, S. xiii; Dieckmann und Schuemer 1998; Bon 2001, S. 255; Szigeti und Davis 2002a, S. 46). Szigeti und Davis beschreiben dieses Problem wie folgt: „Do organizations concentrate on getting the best return on their portfolio as real estate, OR do they aim at boosting their company's productivity and effectiveness? Do they measure success and value by saving real estate cost OR by enhancing core business profitability?" (Szigeti und Davis 2002a, S. 47; Vgl. hierzu auch die Diskussionen bei Williams 2000, S. 103; Clements-Croome 2006, S. 43).

Als Gründe für den fehlenden Abgleich zwischen Büro und Nutzer lassen sich anführen, dass die Reduktionen von Mietflächen und Mietkosten vergleichsweise schnell und kalkulatorisch bzw. bilanzbuchhalterisch ,sichtbar“ ausgeführt werden können. Dagegen lassen sich Einflüsse auf die Produktivität eines Nutzers oder dessen Zufriedenheit nicht ohne weiteres quantifizieren, weil geeignete Messgrößen nur selten erhoben werden und nachhaltige Wirkungen geeigneter Maßnahmen mittelbis langfristig auftreten, wenn sie denn überhaupt eindeutig den Verbesserungen in die Arbeitsumgebung zugeordnet werden können.

Zur Veranschaulichung der Bedeutung einer umfassenderen Betrachtung der Arbeitsumgebung dient obiges Beispiel. Es zeigt im ersten Schritt auf, dass hinsichtlich der Relation ca. $80 \%$ der Kosten eines Arbeitsplatzes auf das Personal entfallen und ca. $10 \%$ auf die Mietkosten bzw. auf das Equipment (siehe Abb. 1). ${ }^{3}$

\footnotetext{
${ }^{2}$ Eine Übersicht zu Publikationen, welche die Beziehungen zwischen Produktivität und einzelnen Aspekten der Arbeitsumgebung aufzeigen, findet sich bei Clements-Croome (2006).

${ }^{3} \mathrm{Vgl}$. ähnliche Ergebnisse für Ermittlungen der Kostenrelationen bei McDougall et al. (2002); Drake (2002); Brill et al. (2001); Williams (2000); Preiser et al. (1988); Moleski und Lang (1986); Goodrich (1986). Die Relationen bleiben über die Jahre offensichtlich stabil.
} 
Tab. 1 Schätzung der durchschnittlichen Kosten für einen Büroarbeitsplatz am Beispiel Banken und Finanzdienstleistungen für den Standort Frankfurt

Bürofläche je Mitarbeiter (Durchschnitt Banken, Finanzdienstleistungen) ${ }^{a}$

$33,3 \mathrm{~m}^{2}$

Miete p. a. (Mietpreis Bankenviertel Frankfurt, Durchschnittspreis 22,4€/m²)

$269 € / \mathrm{m}^{2}$

Mietnebenkosten p. a. (Nebenkosten Frankfurt, klimatisiert: 3,41€/ $\left.\mathrm{m}^{2}\right)^{c}$

$41 € / \mathrm{m}^{2}$

Summe Mietkosten

$10.323 €$

Möblierung (Kosten für gehobene Büroausstattung: $3300 €$; kalk. Abschreibung 5 Jahre) ${ }^{d} \quad 660 €$

Kosten IT p. a. (Median für Banken-Branche) ${ }^{e}$

$8157 €$

Sonstige (Verbrauchkosten u. Umlagen wie Büromaterial, Strom, Empfang usw.) ${ }^{f}$

$875 €$

Summe Equipmentkosten

$9692 €$

Bruttojahresverdienst (Durchschnitt Finanz- $u$. Versicherungsdienstleistungen) ${ }^{g}$

$64.047 €$

Lohnnebenkosten (im Verhältnis zum Bruttoverdienst) ${ }^{h}$

$28 \%$

Summe Personalkosten

$81.980 €$

${ }^{a}$ Vgl. Jones (2009, S. 3). Die Fläche versteht sich als angemietete Gesamtbürofläche, die neben Hauptund Nebennutzfläche auch Flure, Empfangsbereiche, Konferenzräume usw. pro Mitarbeiter enthält

bVl. BNP Paribas Real Estate (2012, S. 25)

'Vgl. Jones (2010, S. 15)

${ }^{d}$ Vgl. Schmetzer und Schwalm (2011, S. 22)

eVgl. Messerschmidt et al. (2008, S. 12)

${ }^{\mathrm{f}}$ Eigene Schätzung

gDurchschnittlicher Bruttojahresverdienst von Vollzeitbeschäftigten im Jahr 2011 für Erbringung von Finanz- und Versicherungsdienstleistungen, ohne Auszubildende, früheres Bundesgebiet einschl. Berlin, vgl. Statistisches Bundesamt; https:/www.destatis.de/DE/ZahlenFakten/GesamtwirtschaftUmwelt/ VerdiensteArbeitskosten/VerdiensteBranchen/Tabellen/Bruttojahresverdienst.html (Zugriff am 02.08.2012)

hVgl. Statistisches Bundesamt; https://www.destatis.de/DE/ZahlenFakten/GesamtwirtschaftUmwelt/ VerdiensteArbeitskosten/ArbeitskostenLohnnebenkosten/EUVergleich/Tabellen/HoeheLohnnebenkosten. html (Zugriff am 02.08.2012)

Als Basis dieser vereinfachten Schätzung dienen die Angaben in obiger Tabelle, welche aus den jeweils angegebenen Quellen exemplarisch für einen Arbeitsplatz im Banken- und Finanzdienstleistungssektor im Frankfurter Bankenviertel entnommen sind (Tab. 1).

Die aufgezeigte Relation führt unmittelbar zu der Fragestellung, ob die Konsequenzen einer angestrebten Reduktion der Mietkosten einen Effekt auf das Personal hervorrufen können, der die monetären Einsparungen durch Einflüsse auf die Produktivität aufhebt. Werden ausgehend von oben genanntem Beispiel 25\% der Hauptnutzfläche ${ }^{4}$ je Arbeitsplatz reduziert $\left(-8,3 \mathrm{~m}^{2}\right)$ und damit ca. $2500 €$ Mietkosten im Jahr gesenkt, dann würde bei Annahme eines negativen Effekts auf die Produktivität des Nutzers von nur 3\%, welcher sich aus der Veränderung des direkten Arbeitsplatzes ergibt, ein kalkulatorischer Verlust auf Basis der Personalkosten von ca. $2500 €$ resultieren. Die Gesamtkosten würden sich überhaupt nicht verändern und der Einspareffekt wäre damit aufgehoben.

\footnotetext{
${ }^{4}$ Die ursprüngliche Fläche bezieht sich auch auf Nebennutzflächen und anteilige Flächen für Flure, Empfang, Konferenz etc., welche nicht direkt, sondern nur kalkulatorisch zugeordnet sind und damit nicht ohne weiteres reduziert werden können.
} 
Als erste Einschränkung in diesem vereinfachten konstruierten Beispiel ist festzuhalten, dass die Effekte weniger auf Personal- bzw. Gesamtkostenbasis zu ermitteln wären, als vielmehr auf Basis der Umsätze oder Gewinne je Mitarbeiter. ${ }^{5}$ Die durchschnittlichen Umsätze pro Mitarbeiter betragen bspw. für die im DAX gelisteten Banken und Versicherungsunternehmen fast $700.000 €$ p. a. ${ }^{6}$

Des Weiteren fehlen in dieser Betrachtung die nachhaltigen Folgewirkungen einer inadäquaten Arbeitsumgebung, die über die Beeinträchtigung der Leistungsfähigkeit hinausgehen. Diese können in Stress ${ }^{7}$ am Arbeitsplatz resultieren, welcher in Unzufriedenheit mit dem Arbeitsplatz, den Kollegen oder dem Arbeitgeber insgesamt führen kann. In dessen Folge sind gesundheitliche Beschwerden, Fehltage oder (innere) Kündigung der Mitarbeiter zu beobachten. Die Anzahl der Arbeitstage, welche durch Abwesenheit der Mitarbeiter verloren gegangen sind, betrugen Mitte der 1980ger Jahre allein in den USA schätzungsweise 550 Mio. Tage pro Jahr (Harris und Associates 1985, S. 2). In Großbritannien wird Anfang der 1990ger Jahre der Verlust an Arbeitstagen durch Krankheit auf ca. 360 Mio. Tage pro Jahr geschätzt (Sigman 1992, S. 25). In beiden Fällen wird davon ausgegangen, dass die Hälfte der verlorenen Arbeitstage auf Stress am Arbeitsplatz zurückzuführen ist (Elkin und Rosch 1990, S. 741; Cooper et al. 1996, S. 3). Auch die Europäische Kommission beziffert in einer aktuellen Studie, dass $50 \%$ bis $60 \%$ aller Ausfalltage mit Stress in Verbindung stehen (European Commission 2011, S. 6).

Wenn die Arbeitsumgebung auch nur teilweise dazu beitragen kann, diesen Stress am Arbeitsplatz positiv oder negativ zu beeinflussen, dann müssen neben der Relation von Produktivität zu Gewinn oder Umsatz sogar die Folgewirkungen auf Zufriedenheit, Gesundheit, Fehlzeiten oder Fluktuation und die daraus resultierenden Kosten bedacht werden. Als zentrale Problemstellung der vorliegenden Ausarbeitung gilt es deshalb, die Wechselbeziehungen zwischen Immobilie und Nutzer offen zu legen und die Konsequenzen auf Ebene der Mitarbeiter bzw. der gesamten Organisation aufzuzeigen. Wenn sich diese Konsequenzen wie im aufgeführten Beispiel darstellen, wird die zentrale Bedeutung einer adäquaten Büroumgebung für die Nutzer einerseits und den Erfolg eines Unternehmens bzw. einer Organisation andererseits offensichtlich.

\section{Qualitatives Assessment von Büroumgebungen}

Die eingangs formulierte Problemstellung einer nutzerorientierten Bewertung zielt auf die Überprüfung der Eigenschaften einer Büroimmobilie unter Beachtung der

\footnotetext{
${ }^{5}$ Immobilien sind Kosteneinflußgröße und Leistungsparameter. Vgl. Pfnür (2011), S. 223.

${ }^{6}$ Durchschnitt über die Jahre 2004 bis 2011. Quelle: http://www.boerse.de/fundamental-analyse (Zugriff am 02.08.2012) für Allianz SE, Deutsche Bank AG, Deutsche Börse AG, Münchener RückversicherungsGes. AG und eigene Berechnungen. Angaben für Commerzbank AG lagen nicht vor.

${ }^{7}$ Der Begriff Stress als Abstraktion ,... bezeichnet die Summe aller unspezifischen Wirkungen von Faktoren die den Körper beeinflussen können. Diese Agentien werden als ,Stressor' bezeichnet, wenn ihre Fähigkeit, Stress zu erzeugen, gemeint ist.“ Selye (1957). Dabei wirken physiologische und psychologische Determinanten gleichzeitig. ,...Stress is fundamentally a relational concept signifying an imbalance between environmental opportunities and individuals' goals, and capabilities to cope with that imbalance." Evans und Cohen (1991).
} 
Nutzerbedarfe ab. Diese Bedarfe leiten sich aus der Nutzenstiftung einer Immobilie als Ressource ab. Dazu ist eine Bewertung entsprechend der Erfüllung ihrer Zwecke vorzunehmen. Die Betrachtung dieser Zweckerfüllung wird ganz allgemein durch den Begriff der Qualität beschrieben. Sie bedeutet aus interner Sicht die Erfüllung von Standards, Normen, Toleranzen usw., womit sie objektiv messbar ist (Baum et al. 2007, S. 114). Aus externer Sicht umfasst sie „... die Gesamtheit aller Eigenschaften und Methoden eines Produktes oder einer Tätigkeit ..., die sich auf deren Eignung zum Erfüllen gegebener Erfordernisse bezieht.“ (Baum et al. 2007, S. 115). Die Beurteilung einer Immobilie als betriebliche Ressource ist demnach neben der internen, technischen Qualität durch eine Bewertung hinsichtlich der nutzerorientierten zweckbezogenen Eignung vorzunehmen. Insbesondere diese „Fitness for Use“ ist jedoch nur subjektiv durch die Wahrnehmung und Bewertung der Nutzer messbar (Baum et al. 2007, S. 118).

Der klassische Schwerpunkt der Qualitätsbetrachtung einer Immobilie liegt vor allem auf der Entstehungsphase, mit deren Ende ein bauliches Konstrukt in die Nutzung übergeben wird. Die Phase wird flankiert durch gesetzliche Leitlinien, Bestimmungen und Verfahrensvorschriften zu baulicher Nutzung, verwendeter Materialien, Sicherheits- und Gesundheitskoordination (SIGE) auf Baustellen aber auch Anforderungen z. B. für das Einrichten und Betreiben von Arbeitsstätten (Verordnung über Arbeitsstätten, ArbStättV). Letztgenannte Verordnung enthält Mindestvorschriften für Sicherheit und Gesundheitsschutz der Beschäftigten und zielt bei den Arbeitsbedingungen vornehmlich auf gesundheitlich zuträgliche Bedingungen ab. ${ }^{8}$ Die Erfüllung aller Standards und Regularien führt schon aufgrund dieser Einschränkungen nicht automatisch zu einem „guten“ Gebäude (Dieckmann und Schuemer 1998, S. 28; Tan et al. 2002). Darüber hinaus ist jede Immobilie einzigartig. Sie wird bestimmt durch den Standort, die Konstruktion, die Ausstattung aber auch durch die Nutzung und die Eigentumsverhältnisse, weshalb jede für sich als „Prototyp“ anzusehen ist. Die aus technischen Bewertungen gewonnenen Erkenntnisse lassen sich daher nur bedingt auf andere Immobilien übertragen und dürfen mithin auch keinen Anspruch auf die Eigenschaften aus Sicht der Nutzer postulieren. Die Überprüfung der Qualität ist deshalb auf die externe, nutzerorientierte Betrachtung auszuweiten (Wellner 2003, S. 8; Zimmerman und Martin 2001, S. 169; Bordass und Leaman 2005, S. 72; Brill et al. 1984, S. 16).

Instrumente für diese qualitative Beurteilung in der Nutzungsphase einer Immobilie werden unter dem Begriff Post-Occupancy Evaluation zusammengefasst. „Post-occupancy evaluation (POE) is a process of systematically evaluating the performance of buildings after they have been built and occupied for some time. POE differs from other evaluations of building performance in that it focuses on the requirements of building occupants, including health, safety, security, functionality and efficiency, psychological comfort, aesthetic quality, and satisfaction" (Federal Facilities Council 2002, S. 1).

\footnotetext{
${ }^{8}$ Die Arbeitsstättenverordnung dient der Sicherheit und dem Gesundheitsschutz der Beschäftigten beim Einrichten und Betreiben von Arbeitsstätten. § 1 Abs. 1 ArbStättV. Die zugehörigen Technischen Regel für Arbeitsstätten (Arbeitsstättenregeln - ASR) dienen der Unterstützung und Konkretisierung bei der Umsetzung der Schutzmaßnahmen, obgleich eine Verpflichtung zur Anwendung aus ArbStättV nicht hervorgeht.
} 
In jüngerer Vergangenheit tritt auch der Aspekt der Nachhaltigkeit in den Mittelpunkt der Betrachtung von Immobilien, häufig im Zusammenhang mit dem Begriff des ,green building“‘. Dabei wird die Immobilie ebenfalls als Ressource in den Vordergrund gestellt, allerdings aus ökologischen Gesichtspunkten. ${ }^{9}$ Diese Instrumente mit Fokus auf ,green building“ entsprechen hinsichtlich der Verfahrensweise prinzipiell einer POE, aber inhaltlich werden zum Teil andere Zielstellungen verfolgt, welche nicht primär auf den Nutzer ausgerichtet sein müssen (Preiser und Schramm 2012, S. 22 ff.). Ein ökologisch vorbildlich bewertetes Gebäude muss nicht zwangsläufig eine überdurchschnittliche Erfüllung der Nutzeranforderungen erzielen oder automatisch aus Gesichtspunkten der Nutzerzufriedenheit und -produktivität von Vorteil sein, auch wenn diese Beziehung aus marketingorientierten Aspekten mitunter proklamiert wird. ${ }^{10}$

Der Qualitätskreislauf einer Immobilie stellt sich unter Berücksichtigung der zuvor diskutierten Aspekte abschließend wie folgt dar (Abb. 2):

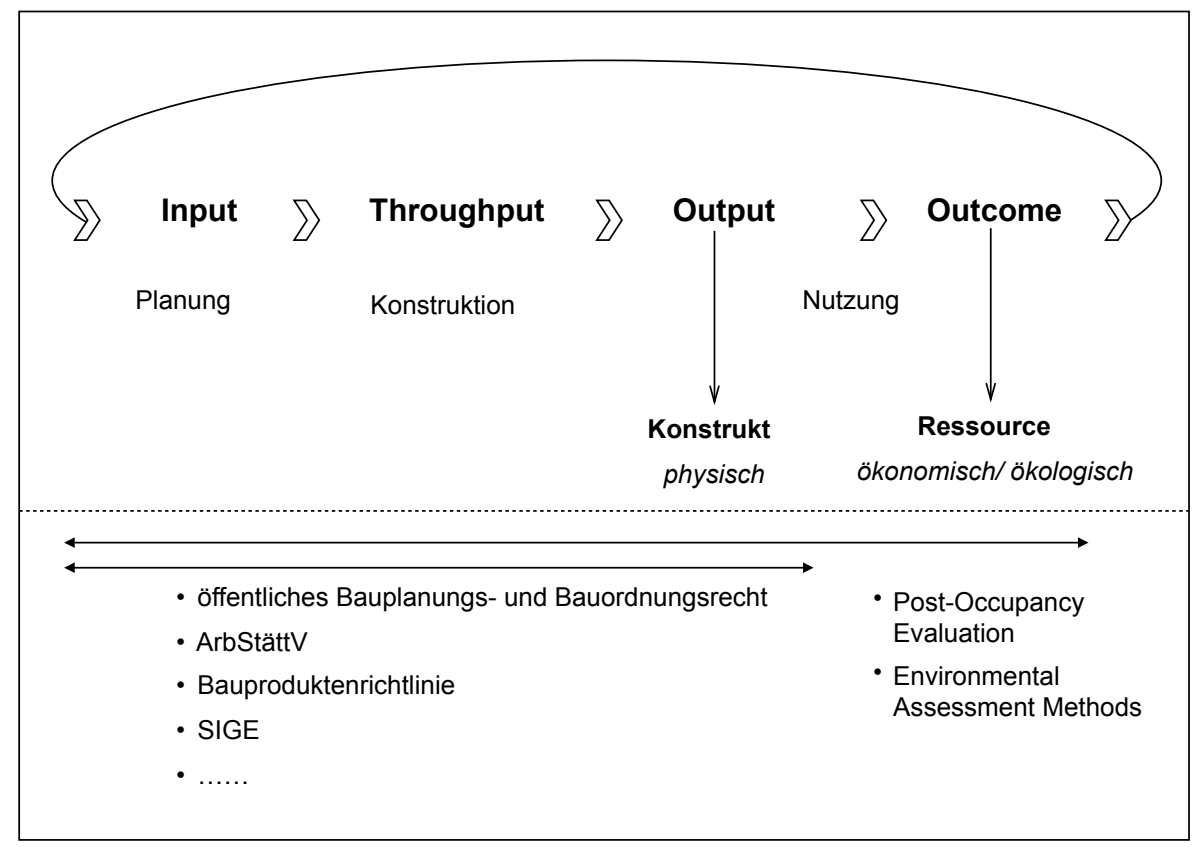

Abb. 2 Qualitätskreislauf im Lebenszyklus der Immobilie

\section{Kriterien zur Evaluation einer Büroimmobilie aus Nutzerperspektive}

Um die Kriterien für die angestrebte Evaluation zu bestimmen, ist zuerst der Bezug zwischen den Umgebungsbedingungen und den menschlichen Reaktionen herzustel-

\footnotetext{
${ }^{9}$ Denkbar sind darüber hinaus auch ästhetische, kulturelle, technische oder funktionale Perspektiven.

${ }^{10}$ Interessant erscheint in diesem Zusammenhang die Einstellung der Nutzer ggü. zertifizierten Immobilien. Einer Untersuchung von Deuble und DeDear zufolge zeigten Nutzer bei deklarierten Green Buildings eine höhere Toleranz hinsichtlich des thermischen Komforts. Vgl. Deuble und deDear (2010).
} 
len. Dabei kann auf die Erkenntnisse des Environmental Behavior zurückgegriffen werden. In dessen Mittelpunkt stehen Erläuterung und Erklärung der „,...Verhaltens- und Erlebensweisen von Nutzern in gebauten Umwelten..." (Dieckmann et al. 1998, S. 47). Die Büroimmobilie repräsentiert in diesem Zusammenhang die gebaute Umwelt und stellt mit ihren Arbeitsplätzen ein spezifisches Setting dar, für das die Wechselbeziehungen untersucht werden sollen.

Die zu beschreibenden Wechselbeziehungen sind wiederum zentraler Gegenstand des Forschungsfeldes der Environmental Psychology/Umweltpsychologie. „Environmental psychology is the study of the molar relationships between behavior and experience and the built and natural environments" (Bell et al. 2001, S. 6). Kern dieser Forschung sind die kausalen Zusammenhänge und Wirkbeziehungen aus den direkten und indirekten Einflüssen der physischen Umwelt auf die menschlichen Emotionen sowie Effekte der physischen Reize auf das Verhalten, z. B. auf die Zufriedenheit, die Arbeitsleistung oder die soziale Interaktion (Mehrabian und Russell 1974, S. 4; Baum et al. 1982, S. 32; Wineman 1982a, S. 258; Sundstrom 1991, S. 734; Flade 1998, S. 3; Bell et al. 2001, S. 435).

Das Environmental Behavior analysiert auf Basis von sogenannten Mensch-Umwelt-Transaktionen die Passung zwischen Mensch und Umwelt (Krampen 1990, S. 207). Als Passung wird dabei generell die Übereinstimmung zwischen verschiedenen Bedürfnissen einer Person oder einer Gruppe und ihrer Umwelt bezeichnet (Dieckmann et al. 1998, S. 71; Klockhaus und Habermann-Morbey 1982, S. 79; Stokols 1982, S. 173; Bell et al. 2001, S. 130). Inhaltlich erfolgt eine Prüfung der Unterstützung bzw. Beeinträchtigung des im Setting beabsichtigten Verhaltens (Dieckmann et al. 1998, S. 71). Aus dieser Prüfung lassen sich Rückschlüsse ziehen, welche Variablen mit welcher Ausprägung anzupassen sind, um die Übereinstimmung der Merkmale zwischen Mensch und Umwelt zu erreichen (Kahana 1982, S. 98). Das Nichterreichen der Passung kann, wie oben schon vermutet, zu Stress und Unzufriedenheit führen (Bell et al. 2001, S. 98). Janis konstatiert, dass unter Stress auch das Entscheidungsverhalten beeinflusst wird. Studien zufolge werden demnach in ,stressigen" Situationen Entscheidungen getroffen, noch bevor alle relevanten Informationen Berücksichtigung finden (Janis und Mann 1977, S. 46 ff.; Janis 1993, S. 59).

Die Kriterien einer nutzerbasierten Bewertung von Büroimmobilien lassen sich folglich aus den Erkenntnissen zu den Mensch-Umwelt-Transaktionen der umweltpsychologischen Forschung ableiten. Hierbei wird unterschieden nach: ${ }^{11}$

- Physischen Umweltstressoren: Licht, Raumklima, Raumluftqualität, Lärm und Umweltkontrolle

- Räumlichen Verhältnissen: Privatheit inklusive persönlicher Raum, Territorialität, Dichte und Beengung, Gebäudekonfiguration und Kommunikation

- Ambiente: Ästhetik als Form des Gefallens, Präferenz als Form des Gefallens sowie Repräsentation durch Symbolik

- Standortfaktoren von Immobilien: Lage und Anbindung von Immobilien

\footnotetext{
${ }^{11} \mathrm{Zu}$ den diskutierten Konzepten siehe Vgl. Dieckmann et al. (1998); sowie die Ausführungen in Bell et al. (2001); Sundstrom und Sundstrom (1986); Evans (1982); Brill et al. (1984); Gifford (2007); Stokols und Altman (1991).
} 
Neben der Bedeutung der physischen Umgebungsbedingungen ist aus Sicht des Environmental Behavior zusätzlich die Integration der individuellen und sozialen bzw. organisationalen Situation unabdingbar, um das spezifische Verhalten im Setting zu berücksichtigen (Craik 1973, S. 405 f.; Moos 1973, S. 658; Preiser und Vischer 2005, S. 11). Eine Untersuchung von Stone führte in diesem Zusammenhang zum Ergebnis, dass die Art der Aufgabe einen entscheidenden Einfluss auf das Empfinden diverser Faktoren wie Motivation, Stress, Ermüdung, Entspannung oder architektonische Wahrnehmung hat (Stone 1998, S. 316). „Die Komplexität einer realen Umgebung erschwert die Einschätzung des Einflusses einzelner Parameter auf die menschliche Leistungsfähigkeit, weil viele dieser Parameter gleichzeitig vorhanden sind und deshalb auch gemeinsamen Einfluss auf jede Person ausüben. Darüber hinaus beeinflusst die Motivation der Beschäftigten das Verhältnis zwischen Leistung und Umgebungsbedingungen“" (Olesen 2008, S. 426). Die Evaluation muss deshalb eine Vielzahl an Attributen eines Settings berücksichtigen und explizit neben den physischen auch die sozialen Aspekte einbeziehen (Kahana 1982, S. 98; Dieckmann et al. 1998, S. 71).

Das Modell, das diese verschiedenen Faktoren explizit berücksichtigt, ist das Effektmodell nach Wineman, welches die unterschiedlichen Zusammenhänge aus der Perspektive eines Büromitarbeiters aufzeigt (Wineman 1982a, S. 257; ähnlich auch Goodrich 1986, S. 114). Neben den Umweltstressoren am Arbeitsplatz finden auch Stressmediatoren und persönliche Charakteristika Berücksichtigung. $\mathrm{Zu}$ den Mediatoren gehören Aspekte, welche die Wahrnehmung und Bewertung der Stressoren beeinflussen, wie z. B. persönliches Anspruchsniveau, Erwartungen, Bedürfnisse und Werte.

Als Treiber für diese Mediatoren lassen sich soziale Rahmenbedingungen und die Arbeitsumgebung aus organisatorischer Sicht bestimmen (Wineman 1982a, S. 269 ff.). Dazu ist besonders die Relation zwischen den physiologischen und psychologischen Arbeitsanforderungen einerseits und der Entscheidungsfreiheit im Rahmen der Ausübung der Tätigkeit andererseits zu berücksichtigen (Karasek 1979, S. 287). Nicht zuletzt ist soziale Interaktion mit Kollegen und Vorgesetzten zu beachten, womit das Maß der Integration des Einzelnen in die Gruppe beschrieben wird, seine Identifikation, die Teilnahme an formeller und informeller Kommunikation und nicht zuletzt die Unterstützung bei der Arbeit durch die anderen (Karasek und Theorell 1990, S. 69 ff.).

Daneben bedingen auch persönliche Eigenschaften, sozioökonomische Faktoren und Erfahrungen die Stressreaktionen (Wineman 1982a, S. 257). Dabei spielen das Alter der Person, der Bildungsgrad, die Statusidentifikation und die Dienstzugehörigkeit eine wesentliche Rolle für die Bewertung der Umweltbedingungen (Vgl. Semmer und Udris 2004, S. 174; Cooper und Marshall 1978, S. 82 ff.; Katz 1978a, S. 213; sowie Katz 1978b, S. 721; Fried et al. 2001, S. 363).

Die Reaktionen auf den Stress beschreiben mentale und physiologische Effekte, die ihrerseits den Outcome, wie z. B. die Zufriedenheit und das Wohlbefinden als Zustandsbeschreibung, bestimmen (Wineman 1982a, S. 256; Gifford 2007, S. 372). Der Outcome umfasst dabei die Menge der physiologischen, kognitiven bzw. affektiven und verhaltensbezogenen Aspekte des Individuums, womit auch ein Bezug zu den o. g. Schlüsselfaktoren wie Produktivität, Gesundheit und Zufriedenheit gegeben ist (McMichael 1978, S. 132). Insbesondere die Zufriedenheit bezieht sich in der 
vorliegenden Betrachtung nicht nur auf die Wahrnehmung der Büroumgebung bzw. der Büroimmobilie, sondern auch auf die Wahrnehmung der Arbeit und des Arbeitgebers selbst.

Wineman trennt in seinem Modell den Stress am Arbeitsplatz vom Stress in anderen Bereichen des Lebens, um die bidirektionale Beziehung zu verdeutlichen, die sich beispielsweise ergibt, wenn familiäre oder finanzielle Probleme auf das Arbeitsleben übergehen oder aber Probleme im Berufsleben auf die privaten Verhältnisse einwirken (Wineman 1982a, S. 258).

Nachdem die modelltheoretischen Zusammenhänge zwischen Nutzer und Immobilie diskutiert wurden, liegt im Folgenden der Fokus auf einer empirischen Überprüfung dieser Aspekte, um die gesamthaften Wirkbeziehungen zwischen Büroimmobilie und Nutzer im Sinne der Passung darstellen und beurteilen zu können.

\section{Empirische Untersuchung}

Aufbauend auf den zuvor diskutierten Erkenntnissen, welche in Abb. 3 als Untersuchungsmodell zusammengefasst sind, werden die identifizierten Einflussparameter der gebauten Umwelt getestet, um deren Effekte auf Wahrnehmung und Bewertung einer Büroumwelt zu bestimmen. Der Einfluss auf die Zufriedenheit mit der Büroumgebung, deren Effekte auf die Produktivität und das gesundheitliche Wohlbefinden stehen dabei stets im Vordergrund der Betrachtung. Darüber hinaus gilt es auch, die identifizierten Stressmediatoren aus der Bürotätigkeit sowie persönliche Charakteristika in die Analyse einzubeziehen, da von ihnen theoretisch ebenfalls ein Einfluss auf die Bewertung ausgeht. Finalisiert wird die Untersuchung durch den Abgleich der Einflüsse zwischen der Bewertung der Büroumgebung und die Zufriedenheit mit der Arbeit.

\subsection{Aufstellung der Hypothesen}

Abgeleitet aus den vorhergehenden Diskussionen lassen sich die Hypothesen im Detail wie folgt formulieren:

Die Zufriedenheit mit der Büroumgebung

- steht in Beziehung mit der Wahrnehmung der Platzverhältnisse,

- steht in Beziehung mit den räumlichen Möglichkeiten zur Kommunikation,

- steht in Beziehung zu den physischen Umgebungsbedingungen Licht, Raumklima, Raumluft, Geräusche und Aussicht,

- steht in Beziehung mit der wahrgenommenen Beeinflussung auf die Produktivität und das gesundheitliche Wohlbefinden,

- steht in Beziehung mit dem Erscheinungsbild des Gebäudes,

- steht in Beziehung zu den Standortfaktoren des Gebäudes.

- steht in Beziehung zur Zufriedenheit mit der Arbeit

Die bis zu dieser Stelle aufgeworfenen Thesen beschreiben die typischen Elemente einer Post-Occupancy Evaluation, mit denen Wahrnehmung und Bewertung einer Büroumgebung erfasst werden. Ergänzt werden diese Fragen um persönliche Cha- 


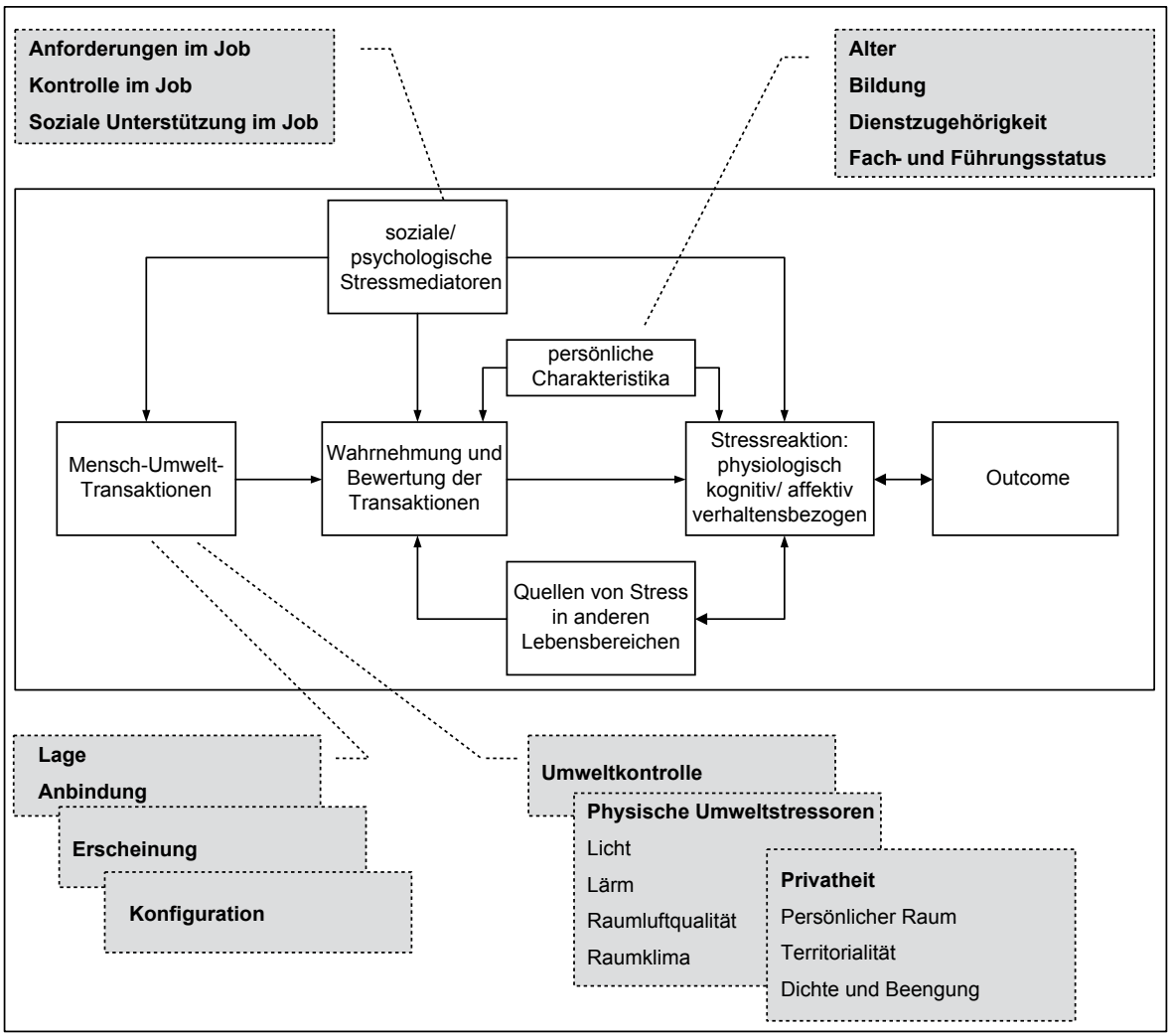

Abb. 3 Einordnung der operationalisierten Aspekte in das Untersuchungsmodell. (Quelle: in Anlehnung an Wineman 1982a. Vgl. hierzu auch McMichael 1978, S. 132)

rakteristika sowie Ausprägungen und Facetten der Tätigkeit, die sozialen Beziehungen und die Aspekte der täglichen Arbeitszeit. Hieraus gilt es folgende Zielstellungen abzufragen:

- Lassen sich Unterschiede in der Wahrnehmung und Bewertung der Büroumgebung anhand der persönlichen Charakteristika nachweisen?

- Lassen sich Unterschiede in der Wahrnehmung und Bewertung der Büroumgebung anhand der Tätigkeitsmerkmale nachweisen?

- Lassen sich Unterschiede in der Wahrnehmung und Bewertung der Büroumgebung aufgrund der Ausprägungen sozialer Beziehungen zu Vorgesetzten bzw. Kollegen nachweisen?

- Lassen sich Unterschiede in der Wahrnehmung und Bewertung der Büroumgebung anhand der zeitlichen Facetten der täglichen Arbeit nachweisen?

\subsection{Auswahl der Untersuchungsobjekte und der Datenerhebungsmethode}

Zur Prüfung der Zusammenhänge wurde eine Erhebung ausgewählter Büronutzer an der Technischen Universität Darmstadt durchgeführt. Als Basis diente eine subjek- 
tive Auswahl von insgesamt 11 Gebäuden mit ca. 1528 potenziellen Büronutzern. ${ }^{12}$ Die Post-Occupancy Evaluation stellt typischerweise eine Felduntersuchung dar, weil sie in der natürlichen (Büro-) Umgebung, also einem realen und keinem kontrollierten Setting, durchgeführt wird. ${ }^{13}$ Für eine leicht handhabbare Erhebung der Daten wurde der schriftlichen Befragung der Vorzug gewährt. Die gewählten Items wurden jeweils anhand subjektiver Wahrnehmung einzelner messbarer Indikatoren von den Teilnehmern erfragt.

Der Rücklauf betrug insgesamt 637 ausgefüllte Fragebögen, was einer Rücklaufquote von ca. 41,7\% entspricht. Die Höhe der Quote zeigt ein reges Interesse der ausgewählten Büronutzer an der Thematik Nutzerzufriedenheit, wobei im Anschreiben zum Fragebogen zusätzlich darauf verwiesen wurde, dass die Ergebnisse der Erhebung den entsprechenden verantwortlichen Institutionen zur Verfügung gestellt werden.

\subsection{Auswertung der erhobenen Daten mittels varianzerklärender Kausalanalyse}

Das Theoriegerüst in Abb. 3, aus dem die Hypothesen abgeleitet sind, zeigt komplexe Strukturen auf, welche nunmehr mit Hilfe eines Strukturgleichungsmodells modelliert und geprüft werden (Homburg et al. 2008, S. 549). Dabei wird auf den varianzanalytischen Ansatz abgezielt, damit die Ausgangsdaten möglichst gut approximiert werden (Chin 1998, S. 301; Weiber und Mühlhaus 2010, S. 58). Dieser Ansatz ist aufgrund des Schätzalgorithmus typischerweise unter der Bezeichnung PLS (Partial Least Squares) bekannt (Homburg et al. 2008). Er ist insbesondere zur Spezifikation formativer Konstrukte geeignet und gilt überdies als robuster bei geringeren methodischen Anforderungen (Knepel 1981, S. 251; Chin 1998, S. 314 f.; Eberl 2004, S. 12; Schloderer et al. 2009, S. 575). Chin/Newstedt empfehlen die Anwendung von PLS u. a. dann, wenn Vorhersagen im Vordergrund stehen, das zu untersuchende Modell relativ komplex ist und mit einer großen Anzahl an Indikatoren bzw. latenten Variablen einhergeht, die Fragestellung relativ neu ist und das theoretische Modell wenig gesichert ist (Chin und Newsted 1999, S. 337).

\subsubsection{Ergebnisse der Schätzung des Kausalmodells für alle Datensätze}

Die umweltpsychologischen Konzepte und sozialen Mediatoren lassen sich mit Hilfe erhobener messbarer Indikatoren als latente Variablen in einem Pfadmodell darstellen. Die Zielgrößen, welche sich aus den Hypothesen ableiten lassen, sind einerseits die „Bewertung der Büroumgebung“ und andererseits die ,generelle Zufriedenheit mit der Arbeit" selbst. Beide latenten Variablen stehen im Mittelpunkt des Modells. Ausgehend von zunächst allen Datensätzen ergeben sich für das Kausalmodell folgende Ergebnisse (Abb. 4):

Die Stärke der Beziehungen zwischen den Konstrukten ist entlang der Pfade als Koeffizient vermerkt. Für die Beziehung, bei denen sich der Koeffizient nicht signi-

\footnotetext{
${ }^{12}$ Die Auswahl erfolgte durch Institutionen der TU Darmstadt.

${ }^{13}$ Vgl. zur Differenzierung zwischen Labor- und Felduntersuchung Bortz und Döring (2006).
} 


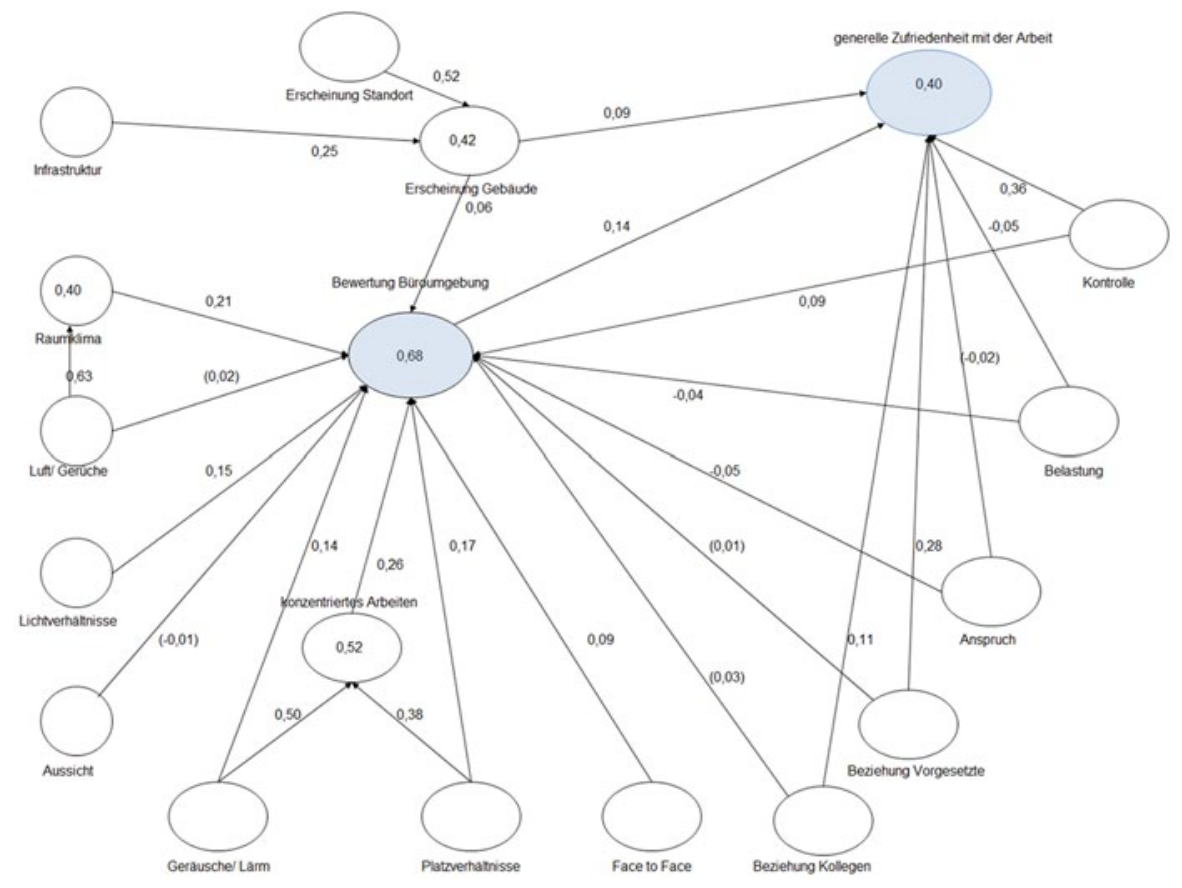

Abb. 4 Ergebnisse des Pfadmodells für alle Datensätze (nicht signifikante Beziehungen $[t$-Wert $<1,65]$ in Klammern dargestellt). (Der Stichprobenumfang entspricht mit $N=637$ mindestens dem 10 fachen der größten Anzahl unabhängiger latenter Variablen (14), welche auf eine abhängige latente Variable (,Büroumgebung“") wirken)

fikant von Null unterscheidet, werden die Ergebnisse in Klammern dargestellt. ${ }^{14}$ Die Bestimmtheitsmaße $R^{2}$, welche innerhalb der Ellipse dargestellt sind, weisen mindestens einen Wert von 0,4 auf und gelten damit als moderat. ${ }^{15}$ Den höchsten Wert kann das Konstrukt „Bewertung Büroumgebung“ mit 0,68 aufweisen, wobei hier der Anteil der erklärten Varianz nach Chin sogar als substantiell zu bezeichnen ist.

Bei Betrachtung der zentralen Beziehung der vorliegenden Untersuchung kann festgehalten werden, dass die Wahrnehmung und Bewertung der Büroumgebung einen signifikanten Einfluss (Koeffizient=0,14) auf die generelle Zufriedenheit mit der Arbeit hat, womit diese Hypothese als Kernaussage des Kausalmodells bestätigt ist. Bei näherer Analyse der Umgebungsaspekte wird ersichtlich, dass Luft/Gerüche und die Aussicht bei paralleler Prüfung aller Umgebungsbedingungen keine relevante Wirkung auf das Empfinden der Büroumgebung ausüben. Allerdings besteht ein signifikanter Effekt von Luft/Gerüche auf das Raumklima, sodass eine Eliminierung der exogenen Variable nicht sinnvoll erscheint.

\footnotetext{
${ }^{14}$ Ausgehend von einer Irrtumswahrscheinlichkeit von $10 \%$ wird der $t$-Wert von 1,65 nicht überschritten.

${ }^{15}$ Chin nennt im Zusammenhang einer konkreten Kausalanalyse ein $R^{2}$ von 0,67 als substantiell, 0,33 als moderat und 0,17 als schwach. Vgl. Chin (1998).
} 
Damit die Einflüsse auf ein Konstrukt insgesamt bewertet werden können, bedarf es einer Betrachtung der totalen Effekte. Diese ergeben sich aus der zusätzlichen Berücksichtigung indirekter Effekte, also über andere Konstrukte vermittelte Effekte, zwischen zwei latenten Variablen, abgeleitet aus der Koeffizientenmatrix (Knepel 1981, S. 263 f.). So ergibt sich der totale Effekt von Luft/Gerüche auf die Büroumgebung aus 0,02 (direkter Effekt) $+0,63 * 0,21$ (indirekter Effekt, der sich aus dem Pfadkoeffizient zum Konstrukt Raumklima und dessen Pfadkoeffizient zu Büroumgebung ergibt) $=0,15$ (totaler Effekt). Auf diese Weise lässt sich der totale Effekt auch für Geräusche/Lärm und Platzverhältnisse mit jeweils 0,27 ermitteln, da diese beiden Konstrukte sowohl direkt als auch indirekt auf die latente Variable Büroumgebung wirken.

Die stärksten Einflüsse auf Wahrnehmung und Bewertung des Büros gehen folglich von den Variablen Geräusche/Lärm, Platzverhältnisse, konzentriertes Arbeiten und Raumklima aus. Geräusche/Lärm und Platzverhältnisse üben ihrerseits einen Einfluss auf die Wahrnehmung und Bewertung der Möglichkeit zum konzentrierten Arbeiten aus. Den Lichtverhältnissen und insbesondere der Möglichkeit zur Kommunikation Face to Face wird hingegen eine geringere bzw. eine zu vernachlässigende Bedeutung beigemessen.

Das Gebäude wird primär durch den Standort geprägt und weniger durch die Infrastruktur, wobei ein Zusammenhang zur Büroumgebung selbst oder der generellen Zufriedenheit mit der Arbeit faktisch nicht gegeben ist.

Die stärkste Wirkung auf das Konstrukt der generellen Zufriedenheit mit der Arbeit ist der Kontrolle zuzuordnen mit einem Koeffizienten von 0,36. Dieses Konstrukt beschreibt die Einflussmöglichkeiten auf die eigene Tätigkeit und die damit verbundenen Freiheitsgrade im Rahmen der täglichen Arbeit. Auch die Beziehung zu den Vorgesetzten erfährt eine hohe Bedeutung mit 0,28, welche zudem stärker ist, als die Beziehung zu den Kollegen. Der Zusammenhang von Belastung und Anspruch aus der eigenen Tätigkeit zur latenten Variable Zufriedenheit mit der Arbeit ist jeweils signifikant negativ, aber aufgrund der absoluten Höhe zu vernachlässigen.

Wird der Einfluss der sozialen Mediatoren auf das Konstrukt Büroumgebung näher beleuchtet, dann muss konstatiert werden, dass die Beziehungen zwar teilweise signifikant, aber ohne nennenswerten Effekt sind.

Die hier vorgestellten Ergebnisse beziehen sich zunächst auf alle Rückläufe der Erhebung. Im folgenden Abschnitt gilt es, das aufgestellte Kausalmodell jeweils anhand von Gruppen zu verifizieren, welche sich aus den Variablen ergeben, die die persönlichen Charakteristika und die Tätigkeitsmerkmale beschreiben. Mit dieser Mehrgruppenkausalanalyse werden Differenzierungen des Modells zwischen Personengruppen untersucht, um mögliche latente heterogene Anforderungen an die Büroumgebung aus den Beurteilungen der Befragten ableiten zu können.

\subsubsection{Ergebnisse der Schätzung des Kausalmodells für unterschiedliche Nutzergruppen}

Als Basis der nachstehenden Betrachtungen dient wiederum das aufgestellte Kausalmodell, welches im vorhergehenden Kapitel systematisch formuliert und geprüft wurde. Im Folgenden werden allerdings die für die Schätzung integrierten Fälle auf 
Tab. 2 Clusterlösungen für persönliche Merkmale

\begin{tabular}{llllll}
\hline $\begin{array}{l}\text { Personen- } \\
\text { Cluster }\end{array}$ & Ausbildung & $\begin{array}{l}\varnothing \text { Jahre in jetzi- } \\
\text { ger Position }\end{array}$ & $\begin{array}{l}\text { Fach-karriere- } \\
\text { stufe }^{\mathrm{a}}\end{array}$ & $\begin{array}{l}\text { Führungs-karrie- } \\
\text { restufe }^{\mathrm{a}}\end{array}$ & $N$ \\
\hline 1 & Berufsschule/Lehre & 8,9 & 2,3 & 1,8 & 94 \\
2 & Uni/Hochschule & 2,1 & 1,7 & 1,4 & 171 \\
3 & Uni/Hochschule & 4 & 3,6 & 3,1 & 261 \\
\hline
\end{tabular}

${ }^{\mathrm{a}} 1$ für unterste Stufe/5 für höchste Stufe

Tab. 3 Clusterlösungen für arbeitszeitliche Merkmale

\begin{tabular}{|c|c|c|c|c|c|}
\hline $\begin{array}{l}\text { Zeit- } \\
\text { Cluster }\end{array}$ & $\begin{array}{l}\text { Anteil Arbeitszeit } \\
\text { am Arbeitsplatz }\end{array}$ & $\begin{array}{l}\text { Zeitanteil } \\
\text { Computer-arbeit }\end{array}$ & $\begin{array}{l}\text { Häufigkeit in } \\
\text { Besprechungen }\end{array}$ & $\begin{array}{l}\text { Häufigkeiten } \\
\text { dienstlich außerhalb } \\
\text { Gebäude }\end{array}$ & $N$ \\
\hline 1 & Mehr als $80 \%$ & Mehr als $80 \%$ & $\begin{array}{l}\text { Mehrmals im } \\
\text { Monat }\end{array}$ & Selten im Monat & 296 \\
\hline 2 & Mehr als $80 \%$ & Mehr als $80 \%$ & $\begin{array}{l}\text { Mehrmals } \\
\text { wöchentlich }\end{array}$ & Selten im Monat & 203 \\
\hline 3 & Bis $\mathrm{zu} 80 \%$ & Bis $\mathrm{zu} 80 \%$ & $\begin{array}{l}\text { Mehrmals } \\
\text { wöchentlich }\end{array}$ & $\begin{array}{l}\text { Mehrmals } \\
\text { wöchentlich }\end{array}$ & 107 \\
\hline
\end{tabular}

die jeweiligen Ergebnisse von zwei Clusteranalysen reduziert, welche sich aus der Differenzierung der Nutzer nach den persönlichen Merkmalen und den arbeitszeitlichen Merkmalen ergeben.

Eine Clusteranalyse nach den Variablen Ausbildung, durchschnittliche Jahre in jetziger Position, Fachkarrierestufe und Führungskarrierestufe ergibt folgende Ergebnisse (Personen-Cluster) (Tab. 2):

Eine weitere Analyse führt in der Differenzierung nach den arbeitszeitlichen Merkmalen zu folgenden Clusterlösungen (Zeit-Cluster) (Tab. 3):

Jede dieser insgesamt sechs Clusterlösungen wird erneut in das oben aufgezeigte Basismodell geladen und geschätzt. Die Ergebnisse werden im Folgenden aus Gründen der Veranschaulichung und der Vergleichbarkeit nicht mehr als Pfadmodell dargestellt, sondern nur noch deren totalen Effekte.

\subsubsection{Zusammenfassende Ergebnisse zur Beurteilung der Büroumgebung}

Eine zusammenfassende Illustration der totalen Effekte für die verschiedenen Cluster-Gruppen in Abb. 5 und Abb. 6 lässt erkennen, dass die Zufriedenheit mit der Lärmsituation und den Platzverhältnissen faktisch über die Cluster hinweg eine Voraussetzung für eine positive Bewertung der Büroumgebung darstellt. Die Bedeutung der hierauf aufbauenden Zufriedenheit mit der Möglichkeit zum konzentrierten Arbeiten variiert insbesondere bei den Zeit-Clustern gegenüber dem Basismodell mit allen Datensätzen, während sie bei den Personen-Clustern prinzipiell eine ähnlich hohe Bedeutung erfährt. Als ebenso bedeutend ist die Zufriedenheit mit dem Raumklima über die Gruppen hinweg einzustufen. Einzig bei Zeit-Cluster 3, also bei jenen Personen, die sich am wenigsten Büro aufhalten, findet sich aufgrund der nichtsignifikanten Korrelation keine Beziehung zwischen Büroumgebung und Raumklima. Die Bedeutung der Lichtverhältnisse wird ebenfalls deutlich, obwohl insbesondere bei den Zeit-Clustern ein heterogenes Bild der Effekte zu erkennen ist. Die Wahr- 


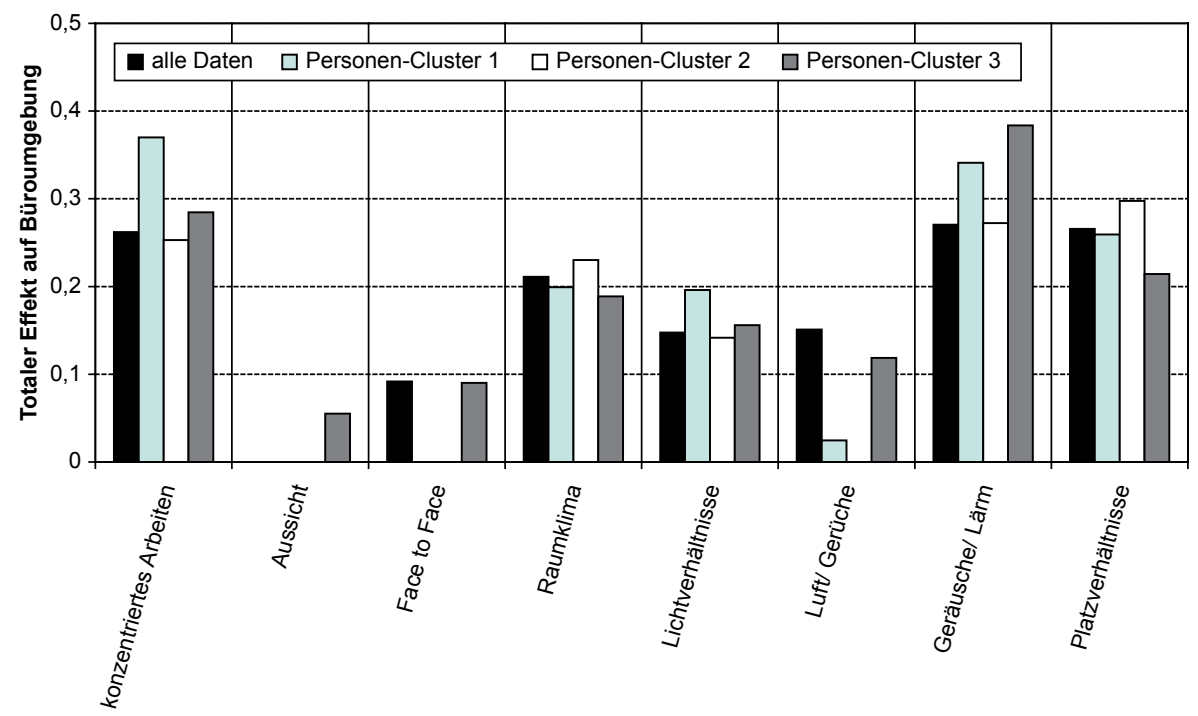

Abb. 5 Totale Effekte der Umgebungsaspekte auf die Büroumgebung für Personen-Cluster

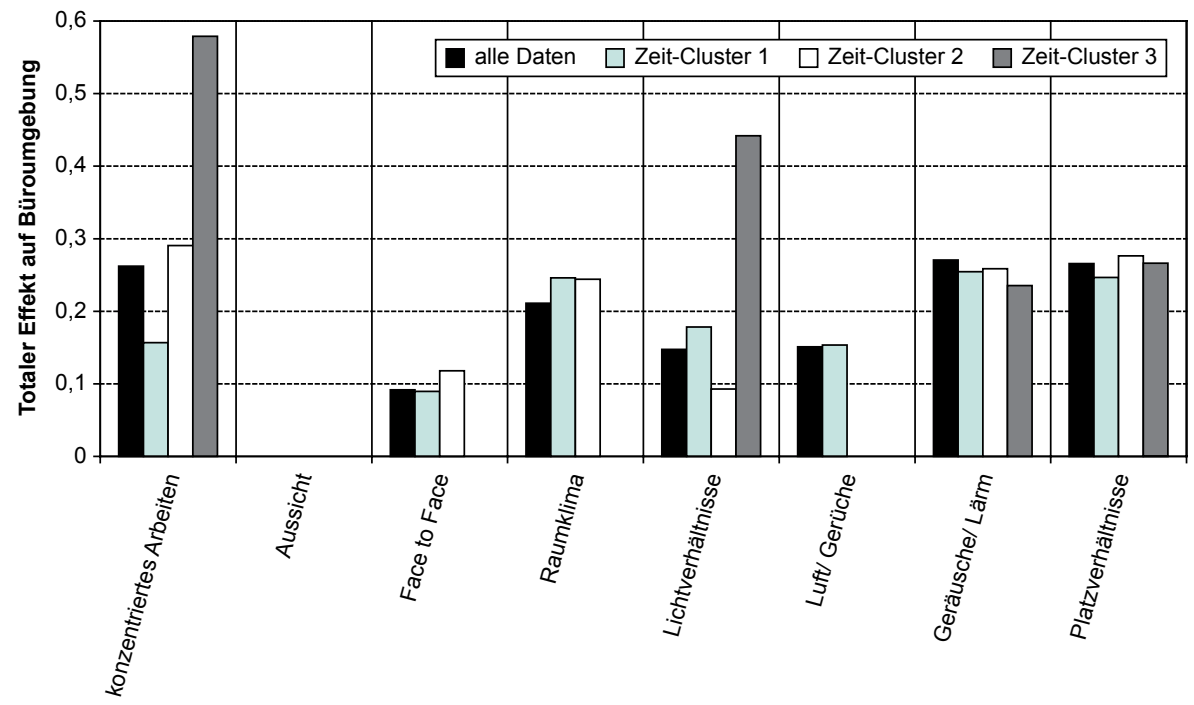

Abb. 6 Totale Effekte der Umgebungsaspekte auf die Büroumgebung für Zeit-Cluster

nehmung und Bewertung von Luft im Zusammenhang mit der Beurteilung der Büroumgebung spielt insgesamt eine geringere Rolle und ist zudem nicht für alle Cluster nachweisbar. Die Relevanz der räumlichen Möglichkeiten zur Kommunikation Face to Face ist ebenso nicht für alle Nutzergruppen nachweisbar. Selbst in den Gruppen, die vergleichsweise häufig ihre Zeit in Besprechungen verbringen, erfährt sie eine geringe Bedeutung. Die Zufriedenheit mit der Aussicht als Einflussfaktor auf die 
Wahrnehmung und Bewertung der Büroumgebung weist nur für Personen-Cluster 3 eine signifikante Relation auf und stellt für die vorliegenden Daten ansonsten keine relevante Variable dar.

\subsubsection{Zusammenfassende Ergebnisse zur Einschätzung der generellen Zufriedenheit mit der Arbeit}

Wird der Fokus der Effekte auf die generelle Zufriedenheit mit der Arbeit gelegt, dann kann aus den Darstellungen in Abb. 7 und Abb. 8 abgeleitet werden, dass die Möglichkeiten der Kontrolle über die Tätigkeiten und Prozesse eines jeden Einzelnen die höchste Bedeutung für die Einschätzung haben, unabhängig von der Zuordnung zu einem Cluster. Den zweithöchsten Einfluss übt die Beziehung zu Vorgesetzten aus, wobei sich dieser Zusammenhang für eine Gruppe (Personen-Cluster 1) erstaunlicherweise gar nicht nachweisen lässt.

Daran schließt sich bereits die Bedeutung der Büroumgebung und teilweise des Gebäudes für die Zufriedenheit mit der Arbeit an. Diese Beziehungen gelten bis auf eine Ausnahme über die verschiedenen Gruppen hinweg. Die Ausnahme bilden die Befragten, welche noch nicht lange in ihrer jetzigen Position tätig sind. Hier kann kein Effekt der Büroumgebung auf die Zufriedenheit nachgewiesen werden, während gleichzeitig die Bedeutung der sozialen Beziehungen eine viel stärkere Rolle gegenüber den anderen Gruppen einnimmt. Die eigenen Einschätzungen von Anspruch und Belastung, welche mit der Tätigkeit in Beziehung gebracht werden, stehen vereinzelt in (negativem) Zusammenhang mit der Arbeitszufriedenheit. Die Infrastruktur und die Beurteilung des Standorts sind hinsichtlich ihrer Bedeutung als vergleichsweise sehr gering, aber dennoch latent wirkend einzustufen, sogar über alle Cluster hinweg.

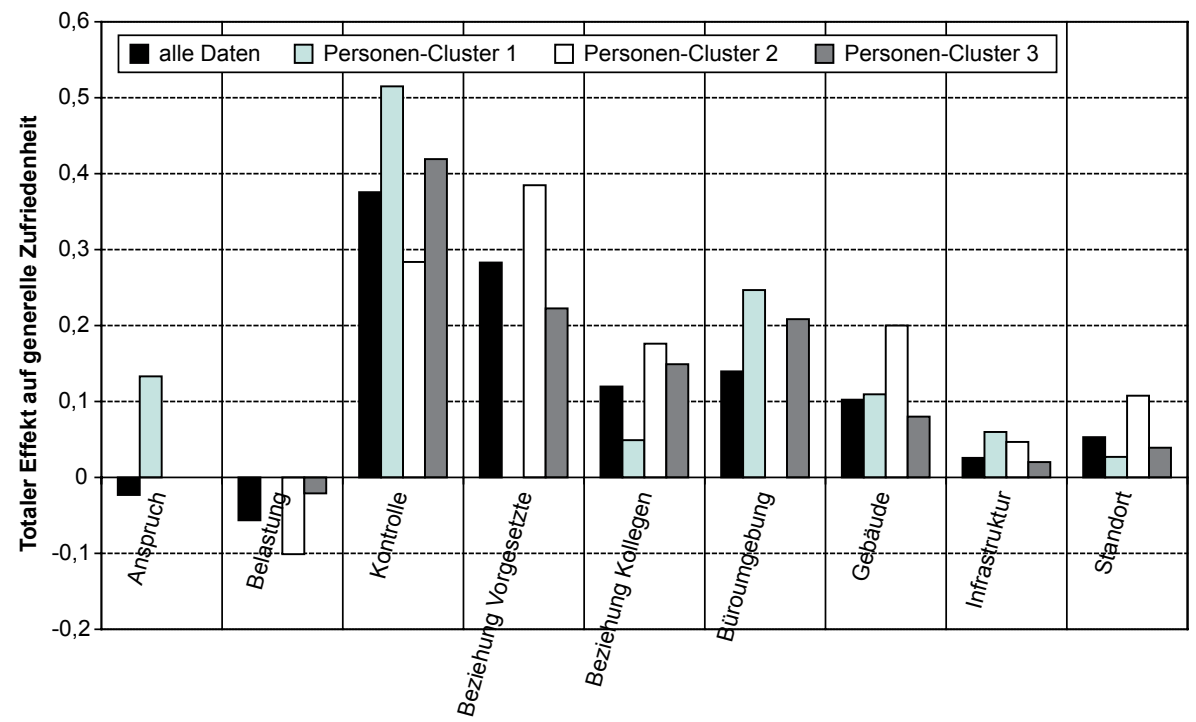

Abb. 7 Totale Effekte auf die generelle Zufriedenheit für Personen-Cluster 


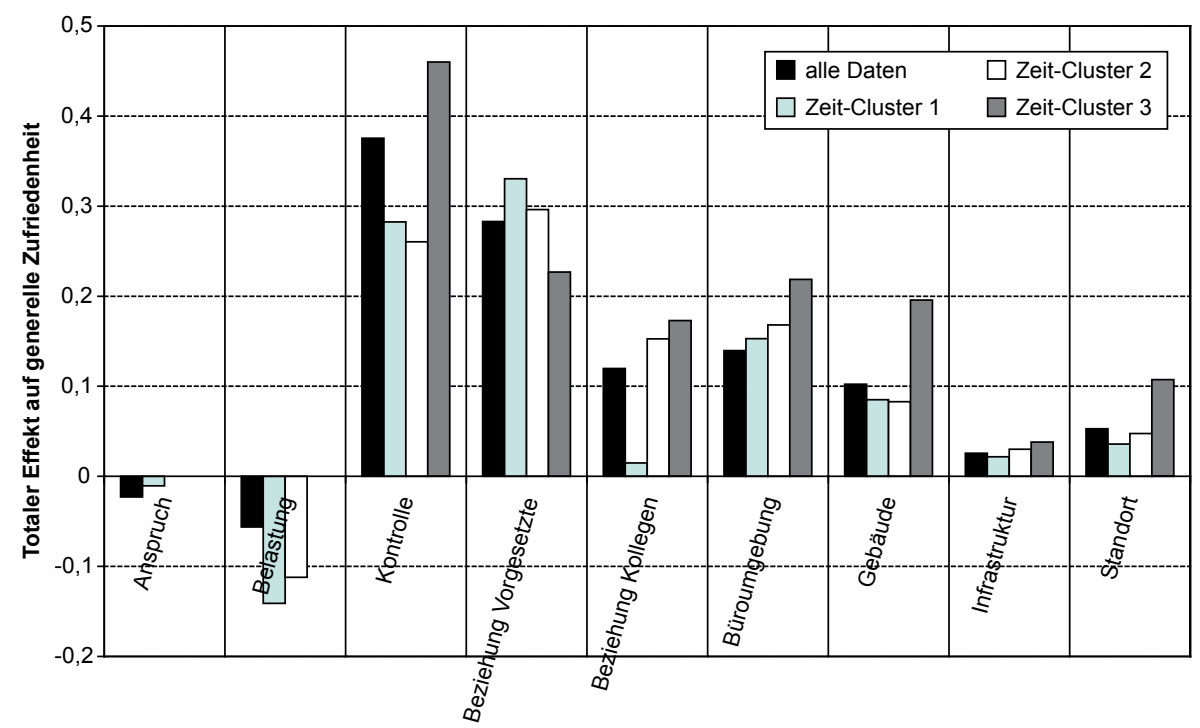

Abb. 8 Totale Effekte auf die generelle Zufriedenheit für Zeit-Cluster

\subsubsection{Bedeutung der Job-Faktoren und der sozialen Faktoren für die Einschätzung der Büroumgebung}

Bei der Betrachtung der totalen Effekte von Job-Faktoren und sozialen Faktoren ist offensichtlich, dass die Aspekte nicht für alle Gruppen einen relevanten Einflussparameter darstellen. Auch sind diese Effekte nicht gleichgerichtet wie die Abb. 9 zeigt. Ein höherer Anspruch der Tätigkeit wirkt insgesamt negativ auf die Einschätzung der Büroumgebung außer bei Personen-Cluster 2. Hier dreht sich die Beziehungsrichtung genauso wie für den Einfluss der Beziehung zu Kollegen bei Zeit-Cluster 2. Die Effekte auf die Büroumgebung sind demnach deutlich heterogener und zugleich schwächer als die der Umgebungsbedingungen, aber dennoch signifikant.

Insgesamt zeigt diese Mehrgruppenkausalanalyse, dass innerhalb der befragten Personen zum Teil erhebliche Wahrnehmungs- und Bewertungsunterschiede in Hinblick auf die Einschätzung einer Büroumgebung auftreten können. Im konkreten Beispiel sind die Nutzer in lediglich jeweils drei Cluster eingeteilt. Dennoch zeigen sich bereits gegenüber dem Basismodell auf Basis aller Datensätze signifikante Unterschiede in der Modellevaluation auf. Eine detaillierte Analyse der Büronutzer wird jedoch mutmaßlich zu einer weiteren Differenzierung einzelner Typen führen und offensichtlich zu einer noch stärkeren Abweichung bei den Einflussparametern. 


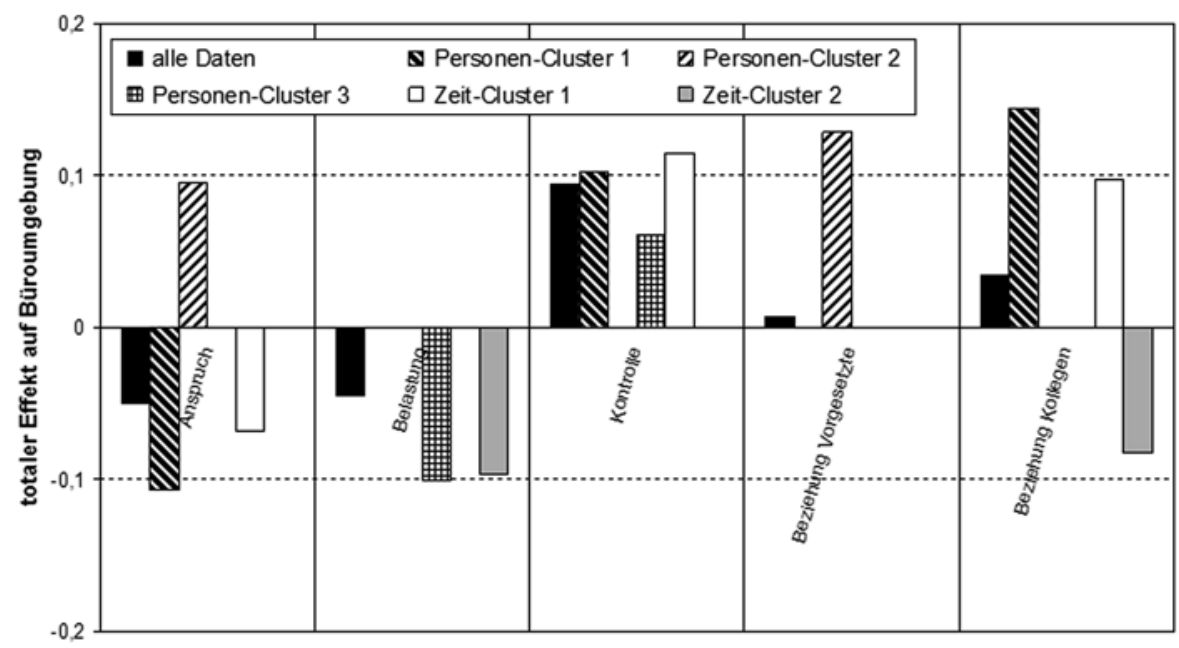

Abb. 9 Totale Effekte der Job-Faktoren und der sozialen Faktoren auf die Büroumgebung für alle Clusterlösungen (Für Zeit-Cluster 3 treten überhaupt keine Effekte auf die Büroumgebung auf)

\section{Post-Occupancy Evaluation als Basis für ein Immobilienportfolio- Benchmarking}

Die Philosophie des Benchmarking beruht auf einem Vergleich von Stärken und Schwächen verschiedener Erfahrungsobjekte (Baum et al. 2007, S. 71). Das Ziel dieses bewertenden Vergleichs ist die Identifikation strategischer Potenziale, die aus der strikten Orientierung an der ,best practice“ abgeleitet werden. Am Beispiel des untersuchten Immobilienportfolios werden die Objekte deshalb hinsichtlich der Einschätzung der Schlüsselfaktoren Zufriedenheit und Produktivität gegeneinander abgetragen und verglichen, wie in Abb. 10 und Abb. 11 auf Basis der erhobenen Daten dargestellt. Die Abbildungen sind getrennt nach Gebäuden als Boxplot abgetragen.

Es lässt sich feststellen, dass der Median über alle betrachteten Immobilien eine zufriedene Ausprägung (oberhalb der neutralen Einschätzung ,weder noch“) aufzeigt. Zwischen den untersuchten Gebäuden bestehen allerdings teils erhebliche Unterschiede. Die beste Einschätzung erhält Gebäude 1, weil selbst der kleinste Wert einer Ausprägung von ,ziemlich zufrieden“ entspricht. Ebenso vorteilhaft erscheinen die Einschätzungen bei den Gebäuden 2, 3, 4, 5 und 7. Dagegen sind bei den Gebäuden mit den Nummern 6,8 und 9 mindestens $25 \%$ der befragten Nutzer unzufrieden (unterhalb der neutralen Einschätzung) mit der Büroumgebung. Auffallend sind auch die stärkeren Streuungen bei den Einschätzungen für die Gebäude 8, 10, 11 und insbesondere bei Gebäude 6 .

Wird dieselbe Betrachtung für die Einschätzung der Einflüsse auf die Produktivität vorgenommen (vgl. Abb. 11), ergibt sich ein differenzierteres Bild. Insgesamt stellt sich die Wahrnehmung über die Einflüsse der Büroumgebung auf die Produktivität negativer dar als die Einschätzung der Zufriedenheit. Für vier der Gebäude wird der Median bei ,bis - 10\%“ ausgewiesen, bei einem weiteren Objekt bei ,bis $-20 \%$ “. Bei den Gebäuden 1 und 4 liegt die Einschätzung von $75 \%$ der Befrag- 


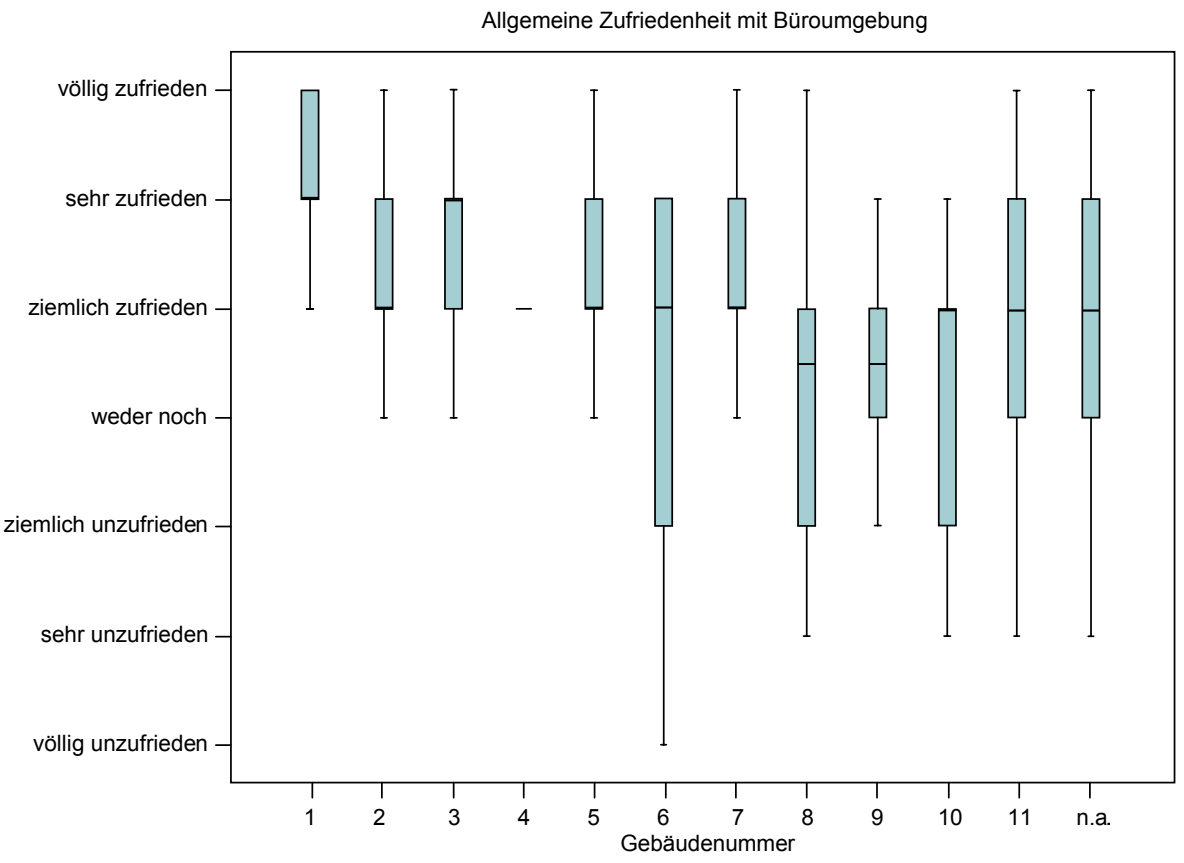

Abb. 10 Vergleich der Einschätzungen zur Zufriedenheit mit der Büroumgebung für das untersuchte Immobilien-Portfolio

Einfluss der Umgebungsbedingungen auf die Produktivität

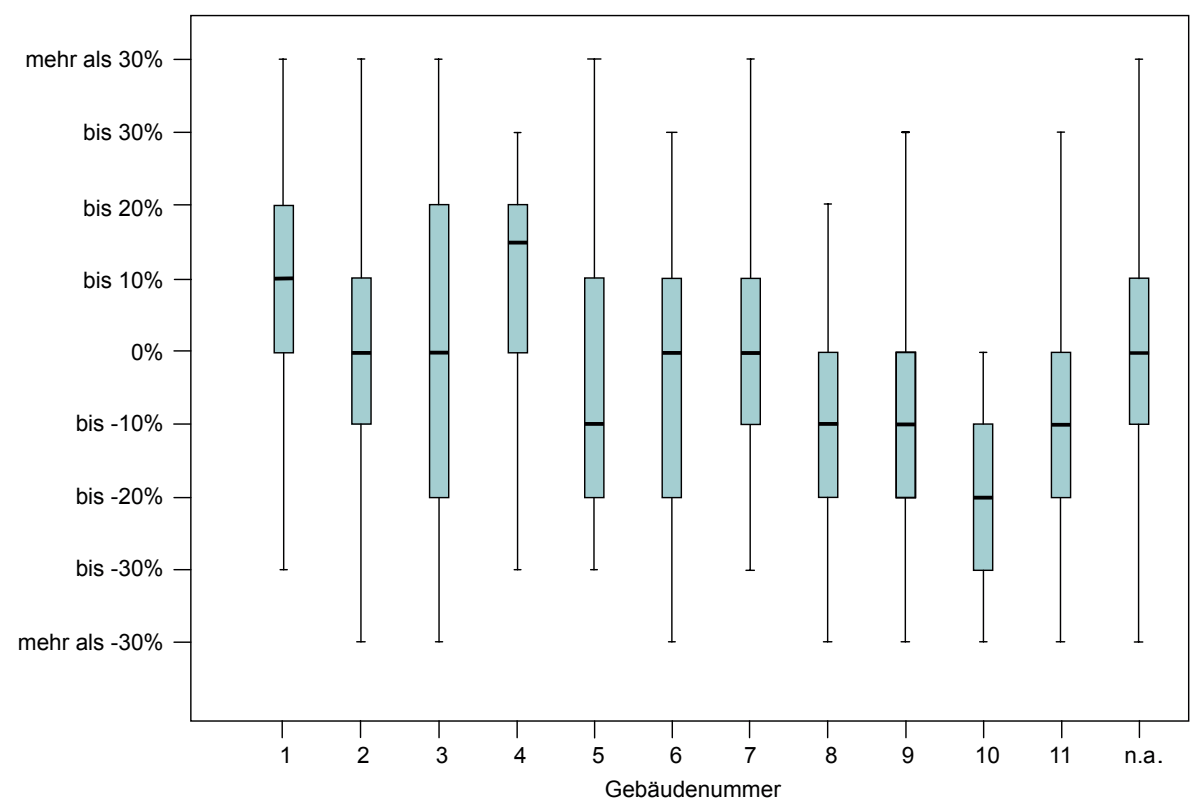

Abb. 11 Vergleich der Einschätzungen zum Einfluss der Umgebungsbedingungen auf die Produktivität für das untersuchte Immobilien-Portfolio 
ten mindestens im neutralen Bereich oder besser, während bei den Gebäuden 8 bis 11 mindestens drei Viertel der Befragten höchstens eine neutrale Einschätzung oder schlechter abgegeben haben. Für Gebäude 5 beträgt dieser Anteil noch immerhin über $50 \%$. Anhand der Daten kann weiterhin für Gebäude 10 konstatiert werden, dass keine positive Beeinflussung der Produktivität durch die Umgebungsbedingungen wahrgenommen wird.

Die größte Spanne zwischen dem $25 \%$ und $75 \%$-Perzentil lässt sich für Gebäude 3 ablesen. In keinem anderen Gebäude ist die Streuung der Einschätzung zum Einfluss der Umgebung auf die Produktivität höher. ${ }^{16}$ Die Gebäude 1 und 4 stellen aus der Sicht der Nutzer offensichtlich positive Beispiele für eine angemessene Büroumgebung dar und definieren damit gleichzeitig die Benchmark, während die mutmaßlich „schlechten“ Objekte 5, 6 und 8 bis 11 einer näheren Untersuchung zu unterziehen sind.

Zur vertiefenden Analyse lässt sich nun im nächsten Schritt auf Basis der bewerteten Items aus der Befragung ein Stammblatt erstellen, welches einen komprimierten Überblick über die einzelnen Aspekte der jeweiligen Immobilie bietet. Diese Auswertung wäre zudem identisch zur Bestandsanalyse bei Betrachtung im Einzelobjektfall.

Nachfolgende Abb. 12 zeigt am Beispiel des Gebäudes 8 eine derartige Übersicht.

Das Stammblatt vermittelt einen Überblick über die wesentlichen Fragen und Antworten der POE, die in Beziehung zum Gebäude und zum Standort stehen. Die Ergebnisse der vorliegenden Befragung sind jeweils als Boxplot horizontal in die Zeilen eingebunden. Der Median ist diesmal aus optischen Gründen als Dreieck dargestellt.

Auffällig ist bei dieser Immobilie insbesondere die Einschätzung zum Raumklima in der Sommerzeit. Drei von vier Büronutzern sind mindestens ziemlich unzufrieden. Die Hälfte aller Befragten ist mit den Gegebenheiten sogar sehr bzw. völlig unzufrieden. Anhand der Einschätzungen der Aspekte Lärm und Luft lassen sich ebenfalls Potenziale zur Verbesserung der Büroumgebung identifizieren. Für alle anderen Aspekte der unmittelbaren Umgebungsbedingungen finden sich dagegen neutrale bis positive Einschätzungen wieder. Im Ergebnis schätzt die Hälfte der Befragten ein, dass ihre Produktivität um mindestens $10 \%$ verringert wird.

In Bezug auf den Standort werden die Angebote für Einzelhandel und Dienstleistungen überwiegend als nicht zufriedenstellend erachtet. Die Imbiss- und Speisemöglichkeiten werden durch die Nutzer im Bereich von ziemlich unzufrieden bis ziemlich zufrieden wahrgenommen. Zudem erfahren das Erscheinungsbild des Gebäudes und dessen Repräsentativität für die Universität eine verbesserungsfähige Bewertung.

Auf Basis dieser Einzelanalyse mit indikativem Evaluationscharakter lassen sich die wesentlichen Aspekte herausstellen, die näher zu überprüfen sind. Für diese vertiefenden Untersuchungsschritte ist nun auf Mittel der investigativen und diagnostischen Evaluation wie technische Messungen, Interviews und Begehungen zurückzugreifen, die der Beschaffung detaillierter Informationen zum status quo dienen. Aus den Ergebnissen dieser weiteren Untersuchungen sind in der Folge mögliche operative Maßnahmen oder organisatorische Handlungsempfehlungen abzuleiten.

\footnotetext{
${ }^{16}$ Hier wäre eine Untersuchung der einzelnen Organisationseinheiten hinsichtlich möglicher Ausstattungsunterschiede in den Büroeinheiten, aber auch bezüglich der Job-Faktoren zur detaillierten Analyse der Spannen angebracht.
} 


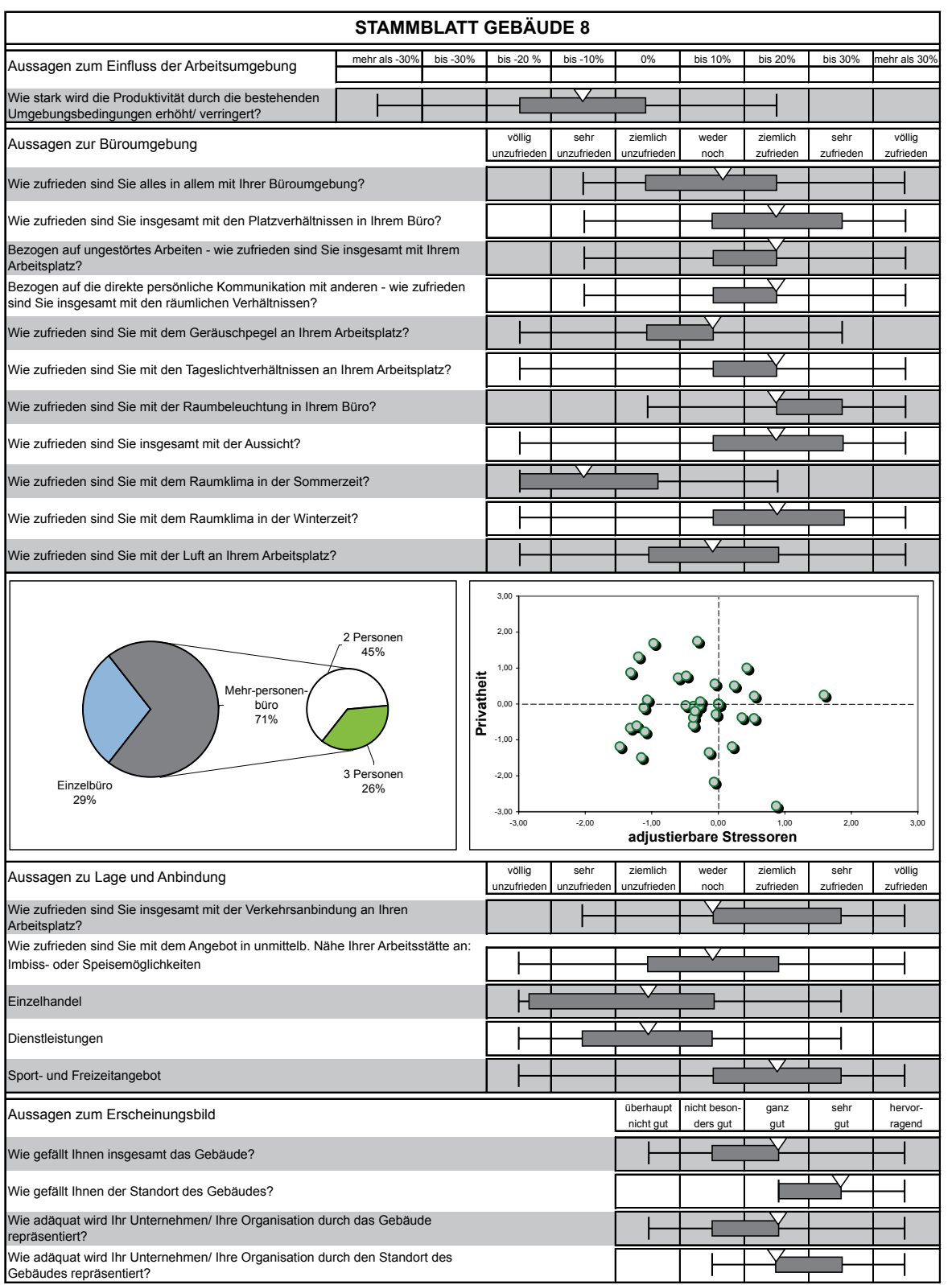

Abb. 12 Stammblatt zu den Ergebnissen der Evaluation für Gebäude 8

\section{Zusammenfassung und Ausblick}

Mit der vorliegenden Ausarbeitung wurde die Zielstellung verfolgt, die Einflüsse einer Büroimmobilie auf den Menschen anhand einer nutzerorientierten Bewertung aufzuzeigen, um Reaktionen und Effekte auf die Erfolgsfaktoren einer Organisation und die Qualität des Arbeitslebens auf individueller Ebene darzulegen. 
Zur Darstellung der diskutierten Zusammenhänge zwischen Mensch und Umwelt wurde eine empirische Untersuchung durchgeführt. Als Ergebnis ist festzuhalten, dass die Befragten im Mittel mit ihrer Büroumgebung zufrieden sind. Allerdings gab die Hälfte der Nutzer einen Einfluss der Umgebungsbedingungen auf die Produktivität im Mittel von bis zu minus $20 \%$ an. Diese Abweichung zu einer neutralen Ausprägung deutet auf eine erhebliche Ineffizienz hin. Hieraus lässt sich wiederum ein enormes Potential zur Steigerung der Leistung sowohl auf Ebene der Mitarbeiter als auch der gesamten Organisation aufzeigen.

Gleichzeitig finden sich aber auch Einschätzungen eines positiven Effekts wieder, wodurch die Bedeutung der Büroumgebung für die Leistungsfähigkeit der Nutzer und der gesamten Organisation unterstrichen wird, auch wenn die hier untersuchte Produktivität der Selbsteinschätzung der Befragten entspricht, die nicht zwangsläufig mit der tatsächlichen bzw. objektiven Leistung übereinstimmen muss.

Anhand der Ergebnisse der Kausalanalyse lässt sich zudem festhalten, dass die Büroumgebung einen signifikant positiven Einfluss auf die Zufriedenheit mit der Arbeit besitzt. Die Einschätzung der Büroumgebung ist dabei teilweise abhängig von den sozialen Beziehungen in den Gruppen und den zeitlichen Belastungen sowie der Entscheidungsfreiheit im Job. Zugleich lässt sich mit der Untersuchung anhand verschiedener Nutzergruppen belegen, dass die Einflüsse der diskutierten Aspekte nicht gleich sind. Daraus kann unmittelbar abgeleitet werden, dass die spezifischen tätigkeitsbedingten und psychosozialen Rahmenbedingungen in die Gestaltung einer optimalen Büroumgebung einfließen müssen. Die Ergebnisse lassen somit auch keine Ableitung der „besten“ bzw. ,richtigen“ Büroumgebung zu.

Anhand der vorgestellten Ausarbeitung und unterstützt durch eine empirische Erhebung lassen sich zusammenfassend folgende Erkenntnisse ableiten:

- Die Büroumgebung übt signifikante Effekte auf Zufriedenheit mit der Arbeit, Leistungsfähigkeit und Gesundheit aus.

- Anhand der Ergebnisse der vorliegenden Studie lassen sich Potentiale zur Steigerung der Produktivität aufzeigen, die durch Verbesserung der Umgebungsbedingungen im Mittel 20\% betragen können.

- Die Zufriedenheit der Nutzer nimmt mit steigendem Grad der Einflussmöglichkeiten auf die Umgebungsbedingungen zu.

- Neben den Umgebungsbedingungen Licht, Lärm, Luft oder Raumklima erfahren die räumlichen Verhältnisse und die Größe des Arbeitsplatzes generell eine mindestens gleichbedeutende Wertung für die Einschätzung der Büroumgebung.

- Unabhängig von den identifizierten Nutzergruppen erfahren die Einschätzung der Lärmsituation, die Einschätzung der Platzverhältnisse im Büro und die Möglichkeit zum konzentrierten Arbeiten eine vergleichsweise hohe Bedeutung für die gesamte Einschätzung der Büroumgebung.

- Die sozialen Verhältnisse zu Kollegen und Vorgesetzten können je nach Nutzergruppe einen signifikanten Einfluss auf die Einschätzung der Büroumgebung ausüben.

- Die wahrgenommene Entscheidungsfreiheit bzw. Kontrolle bei der Arbeit, der Anspruch an die Tätigkeit und die zeitliche Belastung können je nach Nutzer- 
gruppe einen signifikanten Einfluss auf die Einschätzung der Büroumgebung ausüben.

- Je mehr Zeit die Befragten im Büro verbringen, desto kritischer erscheint ihre Wahrnehmung und Bewertung der Umgebungsbedingungen.

- Es besteht keine signifikante Beziehung zwischen der Erfahrung mit unterschiedlichen Bürogebäuden und der Einschätzung der Umgebungsbedingungen.

- Im Vergleich zu allen Umgebungsbedingungen ist die Bedeutung der räumlichen Gegebenheiten für Besprechungen und die Kommunikation Face to Face selbst bei den Nutzern vergleichsweise am geringsten, welche ihre Zeit am häufigsten in Besprechungen verbringen.

- Den Aspekt des Gefallens interpretieren Büronutzer aus ästhetischen Gesichtspunkten und aus Perspektive der Nutzungsfähigkeit gleichermaßen.

- Die Einschätzungen der Nutzer konnten in der vorliegenden Untersuchung durch das Management nicht korrekt wiedergegeben werden. Die Meinungen des Managements zur Einschätzung der Büroumgebung durch die Mitarbeiter stehen in keiner nennenswerten Beziehung. ${ }^{17}$

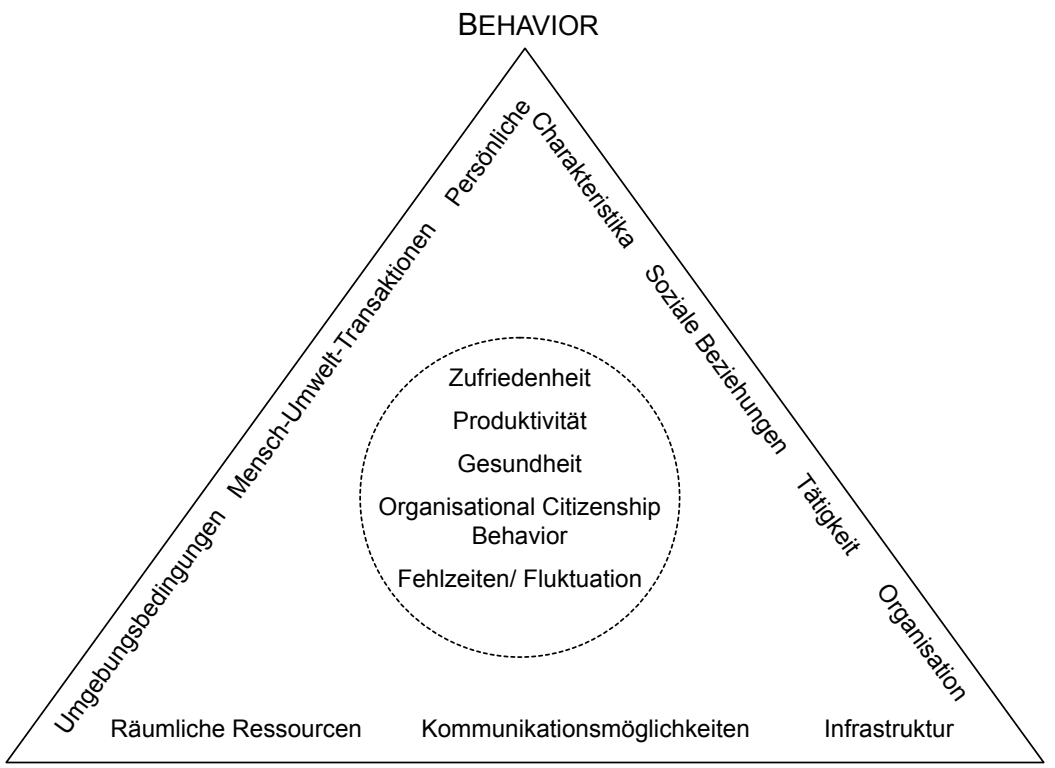

BUILDING

BUSINESS

Abb. 13 Rahmenmodell für die Wechselbeziehungen zwischen Building, Business and Behavior

\footnotetext{
${ }^{17}$ Mit Hilfe einer Datentriangulation wurde der Frage nachgegangen, inwieweit das Management in der Lage ist, die verhaltensbezogenen Antworten aus Sicht der Mitarbeiter korrekt wiedergeben zu können.
} 
Die Untersuchungsergebnisse haben aufgezeigt, dass Wechselbeziehungen zwischen der Immobilie, der Arbeit und dem Verhalten der Nutzer bestehen. Diese Betrachtungsfelder lassen sich auch als Dimensionen eines Rahmenmodells verstehen, welches in nachfolgender Abbildung dargestellt ist (Abb. 13).

Die Gültigkeit dieser gegenseitigen Abhängigkeiten ist offensichtlich für jedes Setting und jede Organisation zu spezifizieren. Eine große Unbekannte bleibt darin der Mensch, denn neben der Individualität ist auch das Verhalten nicht immer getrieben von Rationalität, Wissen oder Bedacht (Berlyne 1975, S. 78).

Für diese Spezifikation erscheint eine Post-Occupancy Evaluation als Instrument geeignet, da sie neben der primär fokussierten nutzerorientierten Bewertung auch als Instrument für die Bestandsanalyse von Portfolios und der Strategieentwicklung im Immobilienmanagement eingesetzt werden kann.

Als Einschränkungen der Untersuchung in Bezug auf die Ableitung allgemeingültiger Aussagen sind insbesondere die spezifischen Verhältnisse der untersuchten Immobilien an der TU Darmstadt zu nennen. Allerdings ist diese objektbezogene Einschränkung in Bezug auf eine nutzerorientierte Bewertung systemimmanent, weswegen der Anspruch der Allgemeingültigkeit der vorliegenden Ergebnisse per se in Frage zu stellen ist. Des Weiteren bleibt die Frage zu klären, ob sich die Büronutzer einer Universität von denen in anderen Organisationen bzw. Unternehmen unterscheiden. Hier ist grundsätzlich die Überlegung anzustellen, dass die Ziele einer Hochschule weniger auf wirtschaftlichen und finanziellen Erfolg ausgerichtet sind, sondern vor allem auf Forschung und Lehre, ermessen an den Ergebnissen in der Forschung und am Ansehen der Einrichtung. Allerdings ist anzunehmen, dass sich Arbeitsalltag, Arbeitsmethodik, Arbeitsanspruch und nicht zuletzt die Verwaltung der Büronutzer wenig von anderen Organisationen unterscheiden, in denen komplexe Aufgabeninhalte den Kern des Handelns bestimmen.

Open Access Dieser Artikel wird unter der Creative Commons Namensnennung 4.0 International Lizenz (http://creativecommons.org/licenses/by/4.0/deed.de) veröffentlicht, welche die uneingeschränkte Nutzung, Verbreitung und Wiedergabe für beliebige Zwecke erlaubt, sofern Sie den/die ursprünglichen Autor(en) und die Quelle ordnungsgemäß nennen, einen Link zur Creative Commons Lizenz beifügen und angeben, ob Änderungen vorgenommen wurden.

\section{Literatur}

Baum, A., Singer, J. E., \& Baum, C. S. (1982). Stress and the environment. In G. W. Evans (Hrsg.), Environmental stress (S. 15-44). Cambridge: Cambridge Univ. Pr.

Baum, H.-G., Coenenberg, A. G., \& Günther, T. (2007). Strategisches controlling (4. üb. Aufl.). Stuttgart: Schäffer-Poeschel.

Bell, P. A., Greene, T. C., Fisher, J. D., \& Baum, A. (2001). Environmental psychology. Belmont: Wadsworth.

Berlyne, D. E. (1975). Behaviourism? Cognitive theory? Humanistic psychology? To Hull with them all. Canadian Psychological Review, 16(2), 69-80.

BNP Paribas Real Estate. (2012). Büromarkt Deutschland. In BNP Paribas Real Estate GmbH (Hrsg.), Property Report, 2012.

Bon, R. (1989). Building as an economic process, an introduction to building economics. Englewood Clliffs: Prentice-Hall. 
Bon, R. (2001). The future of building economics: A note. Construction Management and Economics, 19, 255-258.

Bordass, B., \& Leaman, A. (2005). Phase 5: Occupancy - post-occupancy evaluation. In W. F. E. Preiser \& J. C. Vischer (Hrsg.), Assessing building performance (S. 72-79). Oxford: Elsevier.

Bortz, J., \& Döring, N. (2006). Forschungsmethoden und Evaluation (4. überarb. Aufl.). Heidelberg: Springer.

Brill, M., Margulis, S. T., \& Konar, E. (1984). Using office design to increase productivity (Bd. 1). Buffalo: Workplace Design and Productivity.

Brill, M., Weidemann, S., \& The Bosti Associates. (2001). Disproving widespread myths about workplace design. Jasper: Kimball International.

Bulwien, H., Denk,U., \& Scheffler, R. (2008). Ergebnisse und Schlussfolgerungen aus aktuellen Büroflächenbestandserhebungen in Deutschland; Zeitschrift für Immobilienökonomie, Sonderausgabe 2008: Büroflächenbeschäftigte und Büroflächenbestände in Deutschland.

Carlopio, J. R. (1996). Construct validity of a physical work environment satisfaction questionnaire. Journal of Occupational Health Psychology, 1(3), 330-344.

Chin, W. W. (1998). The partial least squares approach to structural equation modeling. In G. Marcoulides (Hrsg.), Modern methods for business research (S. 295-336). Mahwah: Erlbaum.

Chin, W. W., \& Newsted, P. R. (1999). Structural equation modelling analysis with small samples using partial least squares. In R. H. Hoyle (Hrsg.), Statistical strategies for small sample research (S. 307341). Thousand Oaks: Sage.

Clements-Croome, D. (2006). Indoor environment and productivity. In D. Clements-Croome (Hrsg.), Creating the productive workplace (S. 25-54). London: Taylor \& Francis.

Cooper, C. L., \& Marshall, J. (1978). Sources of managerial and white collar stress. In C. L. Cooper \& R. Payne (Hrsg.), Stress at work (S. 81-105). Chichester: John Wiley \& Sons.

Cooper, C. L., Liukkonen, P., \& Cartwright, S. (1996). Stress prevention in the workplace, assessing the costs and benefits to organizations (S. 3). Luxembourg: Office for Official Publications of the European Communities.

Craik, K. H. (1973). Environmental psychology. Annual Review of Psychology, 24, 402-422.

Deuble, M., \& de Dear, R. (2010). Green occupants for green buildings: The missing link?, Proceedings of Conference: Adapting to Change: New Thinking on Comfort, Cumberland Lodge, Windsor, UK, 9-11 April 2010, o.S.

Dieckmann, F., \& Schuemer, R. (1998). Kommunikation zwischen den beteiligten Gruppen. In F. Dieckmann, A. Flade, R. Schuemer, G. Ströhlein \& R. Walden (Hrsg.), Psychologie und gebaute Umwelt - Konzepte, Methoden, Anwendungsbeispiele (S. 27-43). Darmstadt: Institut Wohnen und Umwelt.

Dieckmann, F., Flade, A., Schuemer, R., Ströhlein, G., \& Walden, R. (1998). Umweltpsychologische Aspekte. In F. Dieckmann, A. Flade, R. Schuemer, G. Ströhlein, \& R. Walden (Hrsg.), Psychologie und gebaute Umwelt - Konzepte, Methoden, Anwendungsbeispiele (S. 47-73). Darmstadt: Institut Wohnen und Umwelt.

Drake, A. (2002). Moving forward: Beyond cost per square foot-the other critical success factors in workplace change projects. Journal of Corporate Real Estate, 4(2), 160-168.

Eberl, M. (2004). Formative und reflektive Indikatoren im Forschungsprozess: Entscheidungsregeln und die Dominanz des reflektiven Modell. In: Schriften zur Empirischen Forschung und Quantitativen Unternehmensplanung, Heft 19; München.

Elkin, A. J., \& Rosch, P. J. (1990). Promoting mental health at the workplace: the prevention side of stress management. Occupational Medicine: State of the Art Reviews, 5(4), 739-754.

European Commission. (2011). Report on the implementation of the European social partners' framework agreement on work-related stress. Commission staff working paper. Brüssel, 24.02.2011, S. 6.

Evans, G. W. (Hrsg.). (1982). Environmental stress. Cambridge: Cambridge Univ. Pr.

Evans, G. W., \& Cohen, S. (1991). Environmental Stress. In D. Stokols \& I. Altman (Hrsg.), Handbook of environmental psychology (Bd. 1). Malabar: Krieger.

Federal Facilities Council. (2002). Learning from our buildings, a state-of-the-practice summary of postoccupancy evaluation (S. 1). Washington DC.: National Academy Press.

Feige, A., Wallbaum, H., Janser, M., \& Windlinger, L. (2013). Impact of sustainable office buildings on occupant's comfort and productivity. Journal of Corporate Real Estate, 15(1), 7-34.

Ferguson, G. S., \& Weisman, G. D. (1986). Alternative approaches to the assessment of employee satisfaction with the office environment. In J. D. Wineman (Hrsg.), Behavioral issues in office design (S. 85-108). New York: van Nostrand Reinhold. 
Flade, A. (1998). Einleitung. In F. Dieckmann, A. Flade, R. Schuemer, G. Ströhlein, \& R. Walden (Hrsg.), Psychologie und gebaute Umwelt - Konzepte, Methoden, Anwendungsbeispiele (S. 3-24). Darmstadt: Institut Wohnen und Umwelt.

Fried, Y., Slowik, L. H., Ben-David, H. A., \& Tiegs, R. B. (2001). Exploring the relationship between workspace density and employee attitudinal reactions: An integrative model. Journal of Occupational \& Organizational Psychology, 74, 359-372.

Gifford, R. (2007). Environmental psychology, principles and practice (4. Aufl., S. 372). Colville: Optimal Books.

Goodrich, R. (1986). The perceived office: The office environment as experienced by its users. In J. D. Wineman (Hrsg.), Behavioral issues in office design (S. 109-133). New York: van Nostrand Reinhold.

Harris, L., \& Associates. (1985). Poll conducted for metropolitan life foundation. Zitiert in: Cooper, C. L., Liukkonen, P., \& Cartwright, S. (1996). Stress prevention in the workplace, assessing the costs and benefits to organizations. Luxembourg: Office for Official Publications of the European Communities, 1996.

Homburg, C., Pflesser, C., \& Klarmann, M. (2008). Strukturgleichungsmodelle mit latenten Variablen: Kausalanalyse. In A. Herrmann, C. Homburg, \& M. Klarmann (Hrsg.), Handbuch Marktforschung: Methoden - Anwendungen - Praxisbeispiele (3., vollst. überarb. und erw. Aufl., S. 547-577). Wiesbaden: Gabler.

Janis, I. L. (1993). Decisionmaking under stress. In L. Goldberger \& S. Brenitz (Hrsg.), Handbook of stress. Theoretical and clinical aspects (2. Aufl., S. 56-74). New York: Free Press.

Janis, I. L., \& Mann, L. (1977). Decision making, a psychological analysis of conflict, choise, and commitment. New York: Free Press.

Jones L. L. (2009). Büroflächenkennziffern 2009. Studie zur Belegung von Büroflächen.

Jones L. L. (2010). OSCAR 2010. Büronebenkostenanalyse.

Kahana, E. (1982). A congruence model of person-environment interaction. In M. P. Lawton, P. G. Windley, \& T. O. Byerts (Hrsg.), Aging and the environment, theoretical approaches (S. 97-121). New York: Springer Publishing Company.

Karasek, R. (1979). Job demands, job decision latitude, and mental strain: Implications for job redesign. Administrative Science Quarterly, 24(2), 285-308.

Karasek, R., \& Theorell, T. (1990). Healthy work, stress, productivity, and the reconstruction of working life. New York: Basic Books.

Katz, R. (1978a). Job longevity as a situational factor in job satisfaction. Administrative Science Quarterly, 23, 204-223.

Katz, R. (1978b). The influence of job longevity on employee reactions to task characteristics. Human Relations, 31(8), 703-725.

Klockhaus, R., \& Habermann-Morbey, B. (1982). Entwicklung und Testung theoretisch begründeter Instrumente zur Erhebung von Wohnumgebungszufriedenheit. Zeitschrift für Sozialpsychologie, 13, 79-87.

Knepel, H. (1981). Modelle mit unbeobachtbaren Variablen - Der PLS-Ansatz. Statistical Papers, 22(4), 248-279.

Krampen, M. (1990). Umwelteinschätzung (Environmental Assessment). In L. Kruse, C.-F. Graumann, \& E.-D. Lantermann (Hrsg.), Ökologische Psychologie (S. 207-212). München: Psychologie Verlags Union.

Marans, R. W., \& Spreckelmeyer, K. F. (1986). A conceptual model for evaluating work environments. In J. D. Wineman (Hrsg.), Behavioral issues in office design (S. 67-84). New York: van Nostrand Reinhold.

McDougall, G., Kelly, J. R., Hinks, J., \& Bititci, U. S. (2002). A review of the leading performance measurement tools for assessing buildings. Journal of Facilities Management, 1(2), 142-153.

McMichael, A. J. (1978). Personality, behavioural, and situational modifiers of work stressors. In C. L. Cooper \& R. Payne (Hrsg.), Stress at work (S. 127-147). Chichester: John Wiley \& Sons.

Mehrabian, A., \& Russell, J. A. (1974). An approach to environmental psychology. Cambridge: MIT Press.

Messerschmidt, M., Schülein, P., \& Murnleitner, M. (2008). Der Wertbeitrag der IT zum Unternehmenserfolg. Stuttgart: PricewaterhouseCoopers AG WPG.

Moleski, W. H., \& Lang, J. T. (1986). Organizational goals and human needs in office planning. In J. D. Wineman (Hrsg.), Behavioral issues in office design (S. 3-21). New York: van Nostrand Reinhold.

Moos, R. H. (1973). Conceptualizations of human environments. American Psychologist, 28(8), $652-665$. 
Olesen, B. W. (2008). Gefährdet das Raumklima unsere Gesundheit? Neue Erkenntnisse über den Einfluss des Raumklimas auf Gesundheit, Komfort und Leistung. In A. Maas (Hrsg.), Umweltbewusstes Bauen, Energieeffizienz - Behaglichkeit-Materialien (S. 417-432). Stuttgart: Fraunhofer-IRB-Verl.

Pfnür, A. (2011). Modernes Immobilienmanagement (3., vollst. überarb., akt. Aufl.). Heidelberg: Springer.

Pfnür, A., \& Weiland, S. (2010). CREM 2010: Welche Rolle spielt der Nutzer? In A. Pfnür (Hrsg.), Arbeitspapiere zur immobilienwirtschaftlichen Forschung und Praxis, Band Nr. 21.

Preiser, W. F. E., \& Schramm, U. (2012). A process model for building performance evaluation (BPE). In S. Mallory-Hill, W. F. E. Preiser, \& C. Watson (Hrsg.), Enhancing building performance (S. 19-31). Chichester: Wiley-Blackwell.

Preiser, W. F. E., \& Vischer, J. C. (2005). The evolution of building performance evaluation. In W. F. E. Preiser \& J. C. Vischer (Hrsg.), Assessing building performance (S. 3-14). Oxford: Elsevier.

Preiser, W. F. E., Rabinowitz, H. Z., \& White, E. T. (1988). Post-occupancy evaluation. New York: Van Nostrand Reinhold.

Schloderer, M. P., Ringle, C. M., \& Sarstedt, M. (2009). Einführung in die varianzbasierte Strukturgleichungsmodellierung. Grundlagen, Modellevaluation und Interaktionseffekte am Beispiel von SmartPLS. In M. Schwaiger \& A. Meyer (Hrsg.), Theorien und Methoden der Betriebswirtschaft (S. 573-601). München: Vahlen.

Schmetzer, O., \& Schwalm, F. (2011). Flexibilisierungs- und Kostensenkungspotenziale moderner Büroumwelten. In A. Helf \& J. Siegert (Hrsg.), Studie von Excellent Business Center und Ernst \& Young Real Estate GmbH - Oktober 2011.

Selye, H. (1957). Stress beherrscht unser Leben. Düsseldorf: Econ-Verl.

Semmer, N. K. \& Udris, I. (2004). Bedeutung und Wirkung von Arbeit. In H. Schuler (Hrsg.), Lehrbuch Organisationspsychologie (3., vollst. überarb. und erw. Aufl., S. 157-195). Bern: Huber.

Sigman, A. (1992). The state of corporate healthcare. Personnel Management, Feb, 1992, S. 24-31.

Stokols, D. (1982). Environmental psychology: A coming of age. In A. G. Kraut (Hrsg.), The G. Stanley Hall lecture series (Bd. 2, S. 155-206). Washington DC: American Psychological Association.

Stokols, D., \& Altman, I. (Hrsg.). (1991). Handbook of environmental psychology (Bd. 1). Malabar: Krieger.

Stone, N. J. (1998). Windows and environmental cues on performance and mood. Environment and Behavior, 30(3), 306-321.

Sundstrom, E. (1991). Work environments: Offices and factories. In D. Stokols \& I. Altman (Hrsg.), Handbook of environmental psychology, (Bd. 1, S. 733-782). Malabar: Krieger.

Sundstrom, E., \& Sundstrom, M. G. (1986). Work places, the psychology of the physical environment in offices and factories. Cambridge: Cambridge University Press.

Sutton, R. I., \& Rafaeli, A. (1987). Characteristics of work stations as potential occupational stressors. Academy of Management Journal, 30(2), 260-276.

Szigeti, F., \& Davis, G. (2002a). Using the ASTM/ANSI standards on whole building functionality and serviceability for briefing and evaluation. In G. Ang \& M. Prins (Hrsg.), Measurement and management of architectural value in performance-based building (S. 43-61). Rotterdam: CIB Report.

Tan, F., Lee, S., Eang S., \& Ho, D. (2002). A framework for intelligent building classification for commercial buildings in the tropics. In G. Ang \& M. Prins (Hrsg.), Measurement and management of architectural value in performance-based building (S. 161-171). Rotterdam: CIB Report.

Weiber, R., \& Mühlhaus, D. (2010). Strukturgleichungsmodellierung: eine anwendungsorientierte Einführung in die Kausalanalyse mit Hilfe von AMOS, SmartPLS und SPSS. Berlin: Springer.

Wellner, K. (2003). Entwicklung eines Immobilien-Portfolio-Management-Systems: zur Optimierung von Rendite-Risiko-Profilen diversifizierter Immobilien-Portfolios. Leipzig: Inst. für Immobilienmanagement. Norderstedt: Books on Demand GmbH.

Wells, M. M. (2000). Office clutter or meaningful personal displays: the role of office personalization in employee and organizational well-being. Journal of Environmental Psychology, 20, $239-255$.

Williams, B. (2000). An introduction to benchmarking facilities \& justifying the investment in facilities. Bromley: Building Economics Bureau.

Wineman, J. D. (1982a). The office environment as a source of stress. In G. W. Evans (Hrsg.), Environmental stress (S. 256-285). Cambridge: Cambridge Univ. Pr.

Zimmerman, A., \& Martin, M. (2001). Post-occupancy evaluation: Benefits and barriers. Building Research \& Information, 29(2), 168-174. 